\title{
The similarity-aware relational division database operator
}



André dos Santos Gonzaga

\section{The similarity-aware relational division database operator}

Master dissertation submitted to the Instituto de Ciências Matemáticas e de Computação - ICMC-USP, in partial fulfillment of the requirements for the degree of the Master Program in Computer Science and Computational Mathematics. EXAMINATION BOARD PRESENTATION COPY

Concentration Area: Computer Science and Computational Mathematics

Advisor: Prof. Dr. Robson Leonardo Ferreira Cordeiro

USP - São Carlos

June 2017 
Ficha catalográfica elaborada pela Biblioteca Prof. Achille Bassi e Seção Técnica de Informática, ICMC/USP, com os dados fornecidos pelo(a) autor(a)

Gonzaga, André dos Santos
The similarity-aware relational division database
operator / André dos Santos Gonzaga; orientador
Robson Leonardo Ferreira Cordeiro. - São Carlos -
SP, 2017.
70 p.
Dissertação de mestrado (Mestrado - Programa de
Pós-Graduação em Ciências de Computação e Matemática
Computacional) - Instituto de Ciências Matemáticas e
de Computação, Universidade de São Paulo, 2017.
1. Databases. 2. Division in the Relational
Algebra. 3. Comparison by Similarity. 4. Complex
Data. I. Cordeiro, Robson Leonardo Ferreira, orient.
II. Título.


Assinatura:

\section{André dos Santos Gonzaga}

\section{Divisão relacional por similaridade em banco de dados}

Dissertação de mestrado apresentada ao Instituto de Ciências Matemáticas e de Computação - ICMC-USP, como parte dos requisitos para obtenção do título de Mestre em Ciências - Ciências de Computação e Matemática Computacional. EXEMPLAR DE DEFESA

Área de Concentração: Ciências de Computação e Matemática Computacional

Orientador: Prof. Dr. Robson Leonardo Ferreira Cordeiro

\section{USP - São Carlos}

June de 2017 



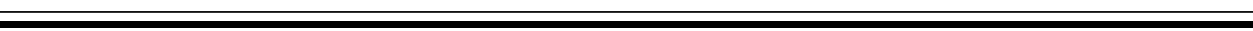

We thank the financial support from FAPESP, projects 2015/05607-6 and 2014/21483-2, CAPES and CNPq. 



\section{RESUMO}

GONZAGA, A. S.. Divisão relacional por similaridade em banco de dados. 2017. $70 \mathrm{f}$. Dissertação de mestrado (Mestrado em Ciências - Ciências de Computação e Matemática Computacional) - Instituto de Ciências Matemáticas e de Computação (ICMC/USP), São Carlos $-\mathrm{SP}$.

O operador de Divisão $(\div)$ da Álgebra Relacional permite representar de forma simples consultas com o conceito de "para todos", e por isso é requerido em diversas aplicações reais. Entretanto, evidencia-se neste trabalho de mestrado que a divisão não atende às necessidades de diversas aplicações atuais, principalmente quando estas analisam dados complexos, como imagens, áudio, textos longos, impressões digitais, entre outros. Analisando o problema verifica-se que a principal limitação é a existência de comparações de valores de atributos intrínsecas à Divisão Relacional, que, por definição, são efetuadas sempre por identidade $(=)$, enquanto objetos complexos devem geralmente ser comparados por similaridade. Hoje, encontram-se na literatura propostas de operadores relacionais com suporte à similaridade de objetos complexos, entretanto, nenhuma trata a Divisão Relacional. Este trabalho de mestrado propõe investigar e estender o operador de Divisão da Álgebra Relacional para melhor adequá-lo às demandas de aplicações atuais, por meio de suporte a comparações de valores de atributos por similaridade. Mostra-se aqui que a Divisão por Similaridade é naturalmente adequada a responder consultas diversas com um conceito de "elementos candidatos e exigências" descrito na monografia, envolvendo dados complexos de aplicações reais de alto impacto, com potencial por exemplo, para apoiar a agricultura, análises de dados genéticos, buscas em bibliotecas digitais, e até mesmo para controlar a qualidade de produtos manufaturados e a identificação de novos clientes em indústrias. Para validar a proposta, propõe-se estudar as duas primeiras aplicações citadas.

Palavras-chave: Bases de Dados, Divisão em Álgebra Relacional, Comparação por Similaridade, Dados Complexos. 



\section{ABSTRACT}

GONZAGA, A. S.. The similarity-aware relational division database operator. 2017. $70 \mathrm{f}$. Master dissertation (Master student Program in Computer Science and Computational Mathematics) - Instituto de Ciências Matemáticas e de Computação (ICMC/USP), São Carlos SP.

In Relational Algebra, the operator Division $(\div)$ is an intuitive tool used to write queries with the concept of "for all", and thus, it is constantly required in real applications. However, as we demonstrate in this MSc work, the division does not support many of the needs common to modern applications, particularly those that involve complex data analysis, such as processing images, audio, genetic data, large graphs, fingerprints, and many other "non-traditional" data types. The main issue is the existence of intrinsic comparisons of attribute values in the operator, which, by definition, are always performed by identity $(=)$, despite the fact that complex data must be compared by similarity. Recent works focus on supporting similarity comparison in relational operators, but no one treats the division. MSc work proposes the new Similarity-aware Division $(\hat{\dot{\div}})$ operator. Our novel operator is naturally well suited to answer queries with an idea of "candidate elements and exigencies" to be performed on complex data from real applications of high-impact. For example, it is potentially useful to support agriculture, genetic analyses, digital library search, and even to help controlling the quality of manufactured products and identifying new clients in industry. We validate our proposal by studying the first two of these applications.

Key-words: Databases, Division in the Relational Algebra, Comparison by Similarity, Complex Data. 

Figure 1.1 - Example of the division used to select cities well suited to produce a particular type of crop. Top: textual tags are compared by identity $(=)$. Bottom: tiles extracted from remote sensing images are compared by similarity $(\hat{=})$. Best viewed in color. . . . . . . . . . . . . . . . . . . 21

Figure 2.1 - Example of the spectrum range of a remote sensing satellite. . . . . . . . 27

Figure 2.2 - An example of SNPs along chromosomes of individuals. . . . . . . . . . 28

Figure 4.1 - Relational Division queries performance on different use cases. . . . . . . . 39

Figure 5.1 - Example of the division used to select cities well suited to produce a particular type of crop. Top: textual tags are compared by identity $(=)$. Bottom: tiles extracted from remote sensing images are compared by similarity $(\hat{=})$. Best viewed in color. . . . . . . . . . . . . . . .

Figure 5.2 - Final setting of our motivational example with cities and crops. The proposed Similarity-aware Division $(\hat{\dot{\div}})$ allows us to select cities well suited to produce a given type of crop, taking as input only georeferenced remote sensing images and city bounds. . . . . . . . . . . . . . . .

Figure 5.3 - Example image with two cities. Its tuple in the dividend CityRegions may be assigned to the group of city A only, since the majority of the image belongs to city A, or to both the groups of city A and city B. It may also be discarded as an outlier tuple. . . . . . . . . . . . . . . 45

Figure 5.4 - Example similar images of water. . . . . . . . . . . . . . . . . 46

Figure 6.1 - Pipeline to preprocess remote sensing images for our case study in agriculture. Best viewed in color. . . . . . . . . . . . . . . . . . . . . 54

Figure 6.2 - The images used in the divisor for the case study in agriculture. They represent the requirements of the crop production. Best viewed in color. . . 54

Figure 6.3 - Example result for two cities. Best viewed in color. . . . . . . . . . . . . 55

Figure 6.4 - Runtime measurements for our algorithms in synthetic data . . . . . . . . . 60 

Table 2.1 - Toy dataset containing genetic data of 3 individuals. . . . . . . . . . . . 28

Table 6.1 - Example of the division used to select animals that satisfy desired genetic conditions, e.g., exceptional milk producers. The dataset represents SNP alleles and their positions along the chromosomes of candidate animals. As opposed to our similarity-aware division, the originaldivision operator fails to identify animal 3, despite the fact that it has SNPs similar to all requirements. 56 

$\hat{\div}-$ Similarity-aware Division

$\hat{=}$ - Similarity between values

$\hat{=}$ - Similarity between tuples

$\hat{\epsilon}$ - Set membership by similarity

$\hat{\subseteq}-$ Subset by similarity

$\hat{-}$ - Difference by similarity

$\mathrm{T}_{G_{k}}$ - Group of similar tuples from a relation

$\mathrm{T}_{G}-$ Set of all groups of similars from a relation 

INTRODUCTION . . . . . . . . . . . . . . . . 19

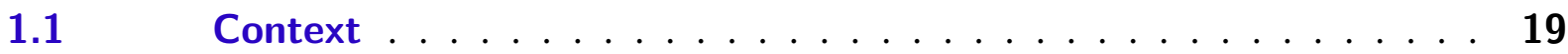

1.2 Problem and Motivation ................. 20

$1.3 \quad$ Contributions . . . . . . . . . . . . . . . . 22

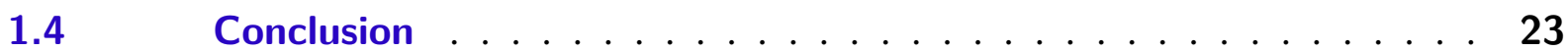

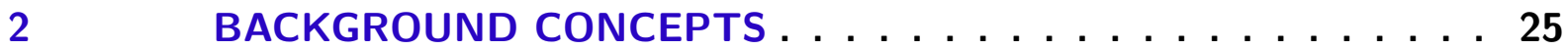

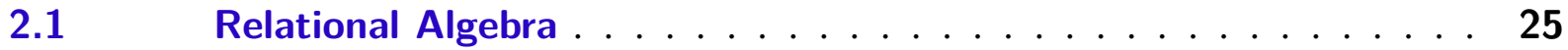

2.2 Relational Division . . . . . . . . . . . . . 25

$2.3 \quad$ Remote Sensing Images . . . . . . . . . . . . . . 27

$2.4 \quad$ Genetic Data . . . . . . . . . . . . . . . . . . 28

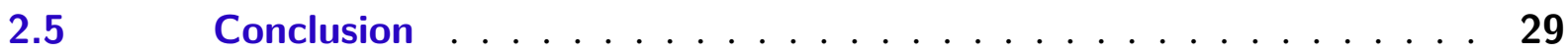

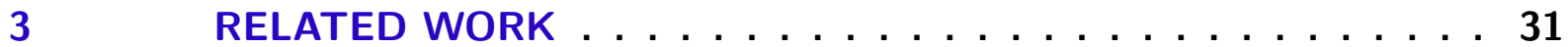

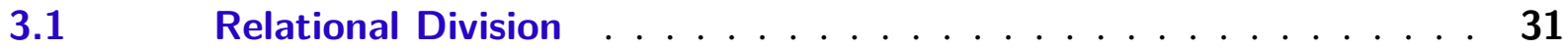

$3.2 \quad$ Similarity-awareness in the Relational Algebra . . . . . . . . . 33

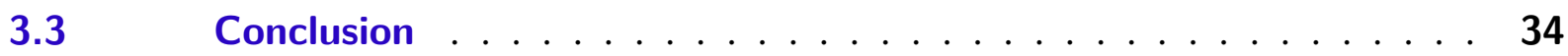

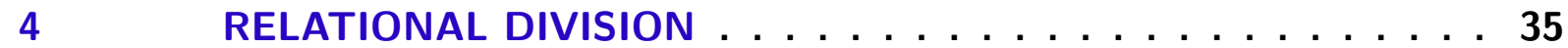

$4.1 \quad$ SQL Implementations for the Division . . . . . . . . . . . . . 35

$4.2 \quad$ Proposed Algorithms . . . . . . . . . . . . . . . 36

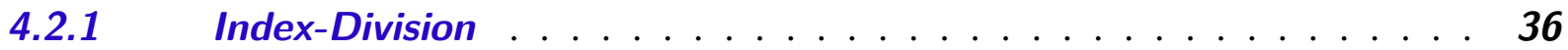

$4.2 .2 \quad$ Division Data Generator . . . . . . . . . . . . . . . . . . . 37

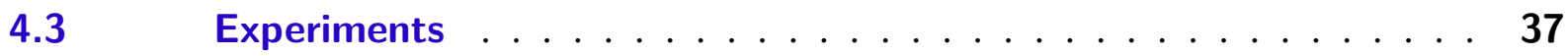

4.3.1 Synthetic Data . . . . . . . . . . . . . . . . . . . 37

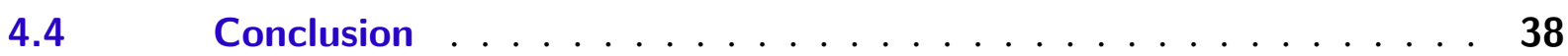

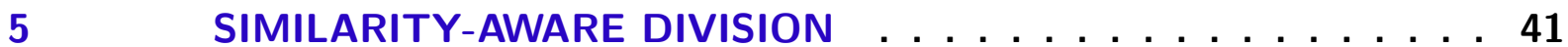

$5.1 \quad$ Proposed Similarity-aware Division Operator . . . . . . . . . . . . 41

5.1.1 Intra and Inter-relation Comparisons . . . . . . . . . . . . . 41

5.1.2 Similarity-awareness in the Division . . . . . . . . . . . . . . 44

5.1.3 Formal Definition . . . . . . . . . . . . . . . . 46

$5.2 \quad$ Proposed Algorithms .................. 48 
EXPERIMENTS . . . . . . . . . . . . . . . . 53

6.0.1 Agricultural Support . . . . . . . . . . . . . . . 53

6.0.2 Selective Animal Breeding . . . . . . . . . . . . . 55

6.0.3 Synthetic Data . . . . . . . . . . . . . . . . . . 58

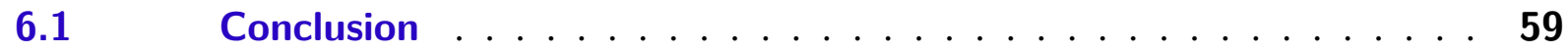

$7 \quad$ DISCUSSION ............................ 61

7.0.1 Automatic Quality Control in Industry . . . . . . . . . . . . . 61

7.0.2 Digital Library Search . . . . . . . . . . . . . . . . . . . . 61

7.0.3 Prospective Client Identification in Enterprises . . . . . . . . . . 62

$7.1 \quad$ Conclusion . . . . . . . . . . . . . . . . . 62

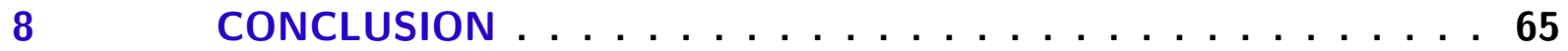

BIBLIOGRAPHY . . . . . . . . . . . . . . . . 67 


\subsection{Context}

The Relational Algebra (CODD, 1972) defines a number of operators to express queries on relations. Among them there are: Projection $(\pi)$, Join $(\bowtie)$, Selection $(\sigma)$, Division $(\div)$, Union $(\cup)$, Intersection $(\cap)$, Difference $(-)$ and Cartesian Product $(\times)$. The Relational Division has an important role in this context because it is the simplest and most intuitive way to represent queries with the idea of "for all", besides being the only algebraic operator that directly corresponds to the Universal Quantification $(\forall)$ from the Relational Calculus (CODD, 1990). As a consequence, the division is required in queries that are commonly performed by real applications. For example, it answers the queries as follows:

- "What students were approved in all Database-related courses?"

- "What products have all requirements of the industrial quality control?"

- "What bank clients paid all bills of their loans?"

- "What cities have all the requirements to produce a given type of crop?"

Let us use the last of these examples to describe the division. The query refers to a problem of "candidate elements and requirements" aimed at identifying cities well-suited to produce a particular type of crop. Figures $1.1 \mathrm{a}, 1.1 \mathrm{~b}$ and $1.1 \mathrm{c}$ illustrate a toy dataset for this query. Relation CityRegions describes three cities, i.e., the candidates, each one represented by a set of regions identified by textual tags. For example, the city of Campinas contains regions with water, urban areas, silos and roads. Relation Requirements describes the needs for the production of a given type of crop. In this particular case, we assume that water, bare soil, silos and urban areas are required. The result of dividing CityRegions by Requirements is relation Cities. It contains the list of cities considered appropriate to produce the crop, that is, those 
cities that have a region tag identical $(=)$ to each tag in Requirements. In this particular case, only the city of São Carlos satisfies all requirements.

\subsection{Problem and Motivation}

Observe that the Relational Division is useful to express complex queries in a simple and intuitive manner, using a single operator. However, we demonstrate in this MSc work that the division cannot properly process "non-traditional" data types that are commonly used in modern applications. The complexity of the data generated or collected by current scientific and commercial applications is increasing in distinct fields, such as biology, physics, medicine, astronomy, climate studies, among others. Today, many real data sets include, besides the traditional numeric values and small texts, more complex data objects such as images, audio files, videos, time series, genetic data elements, large graphs, long texts, fingerprints, and many others (POLA et al., 2013; CORDEIRO; FALOUTSOS; Traina Jr., 2013; ZEZULA et al., 2006; SILVA et al., 2010). One central distinction between traditional and complex data is that the latter must be compared by similarity, since comparisons by identity $(=)$ are in most cases senseless and/or unfeasible for data of a more complex nature (MARRI et al., 2014; MARRI et al., 2016; CORDEIRO; FALOUTSOS; Traina Jr., 2013; JACOX; SAMET, 2008; KALASHNIKOV, 2013; POLA et al., 2015; SILVA et al., 2013; SILVA et al., 2010; TANG et al., 2016a). To illustrate this fact, let us consider again the division query about cities and crops, but now using a more realistic variation of our toy dataset in which we do not have cities carefully partitioned into regions and neither textual tags ready to be used to describe each region. Instead, we have remote sensing images of the cities, and we divide the images into tiles to represent regions, assuming that a specialist user provides any other image or simply picks one of these tiles to represent each requirement. Figures 1.1d, 1.1e and 1.1f illustrate the data. Note that attribute Region has now a complex data type (image) in relations CityRegions and Requirements, but the semantics of both the query and the data remain the same. For example, the city of Campinas still contains regions with water, urban areas, silos and roads, and we still look for cities with the requirements water, bare soil, silos and urban areas. Only the data types were modified. Despite this fact, the Relational Division is now unsuitable to validate the crop's needs, because it is virtually impossible to have any pair of identical (=) image tiles that come from distinct locations. In fact, it is imperative to compare the data by similarity, so to spot distinct - but similar - tiles of the same requirement, such as the tiles of water highlighted with double asterisks in Figures 1.1d and 1.1e. In our notation, we use the symbol $\hat{=}$ to refer to similarity comparison.

Many researchers have been proposing strategies to support similarity comparison in Relational Database Management Systems - RDBMS (SILVA et al., 2010; POLA et al., 2013; BUDÍKOVÁ; BATKO; ZEZULA, 2012; BARIONI et al., 2009; BELOHLAVEK; VYCHODIL, 2010), commonly by means of extending operators of the Relational Algebra. For example, recent works focus on the Join (SILVA et al., 2015; KALASHNIKOV, 2013; SILVA; PEARSON, 2012; 


\begin{tabular}{|c|c|}
\hline City & Region \\
\hline Campinas & Water* \\
\hline Campinas & Urban Area \\
\hline Campinas & Silos \\
\hline Campinas & Road \\
\hline São Carlos & Bare Soil \\
\hline São Carlos & Urban Area \\
\hline São Carlos & Forest \\
\hline São Carlos & Silos \\
\hline São Carlos & Water* \\
\hline Araraquara & Urban Area \\
\hline Araraquara & Bare Soil \\
\hline
\end{tabular}

(a) CityRegions

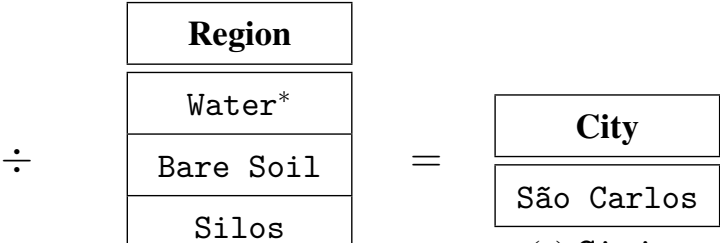 \\ (c) Cities}

* example of identical $(=)$ region tags

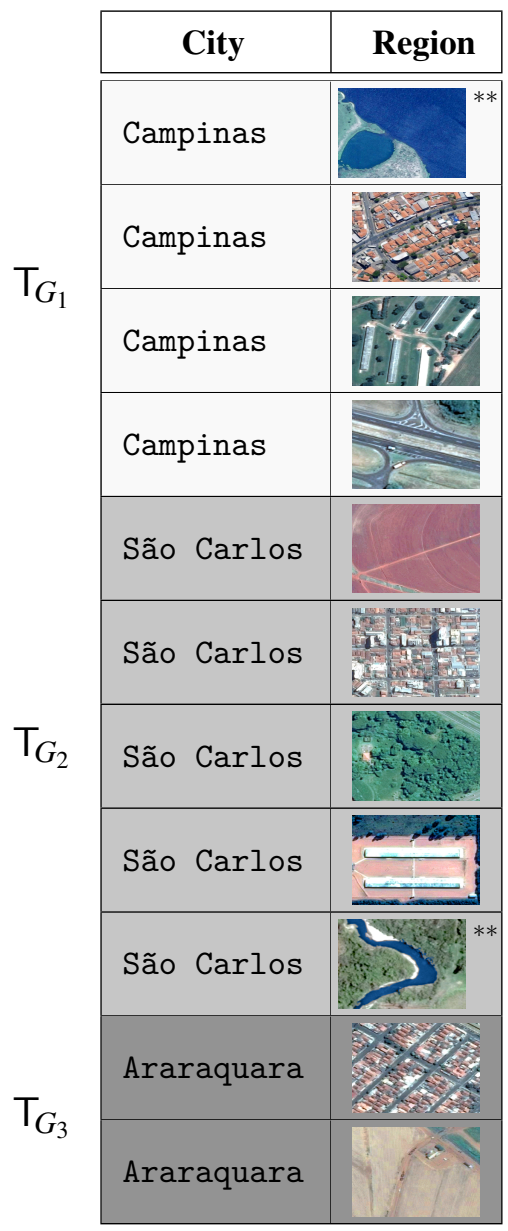

(d) CityRegions

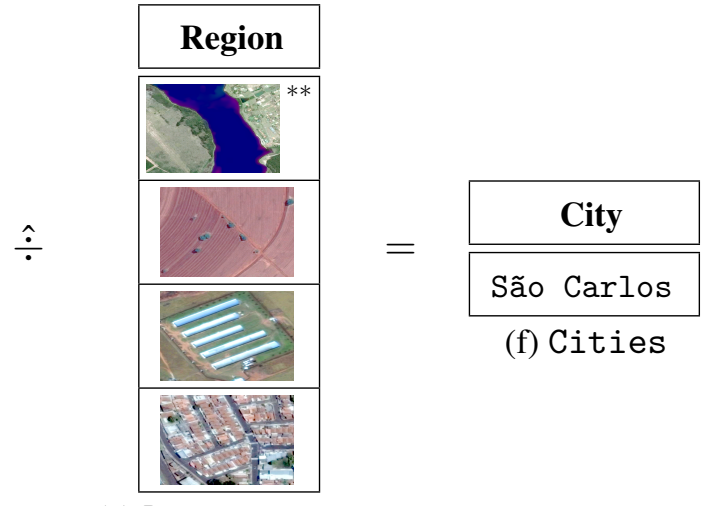

(e) Requirements

** example of similar $(\hat{=})$ tiles of water extracted from remote sensing images

Figure 1.1 - Example of the division used to select cities well suited to produce a particular type of crop. Top: textual tags are compared by identity $(=)$. Bottom: tiles extracted from remote sensing images are compared by similarity $(\hat{=})$. Best viewed in color. 
SILVA; AREF; ALI, 2010), Selection (SILVA et al., 2013; SANTOS et al., 2013), Grouping and Aggregation (TANG et al., 2016a; TANG et al., 2016b; SILVA; AREF; ALI, 2009), Union (POLA et al., 2015; MARRI et al., 2016), Intersection (POLA et al., 2015; MARRI et al., 2014; MARRI et al., 2016) and Difference (POLA et al., 2015; MARRI et al., 2016). However, to the best of our knowledge, no one focuses on the Division. Here, we take the aforementioned works as a premise to explore the hypothesis as follows:

Hypothesis 1: Similarity comparison applied to the Division $(\div)$ database operator turns it into a valuable tool to process complex data coming from modern applications of high-impact.

\subsection{Contributions}

This MSc work investigates Hypothesis 1 focused on querying large sets of complex data. Specifically, we present first Similarity-aware Division $(\hat{\dot{\div}})$ database operator. Our main contributions are:

1. Operator Design and Usability - we identified severe limitations on the usability of the Relational Division to process complex data, and extended it into a new operator to tackle the problem. Our Similarity-aware Division $(\hat{\dot{\div}})$ is naturally well suited to answer queries with an idea of "candidate elements and exigencies" to be performed on complex data from real applications of high-impact. For example, it may support genetic analyses, agriculture, digital library search, and even help controlling the quality of manufactured products and identifying new clients in industry;

2. Formal Definition and Algorithms - we formally defined the similarity-aware division, enabling its usage in queries along with the existing algebraic operators. Also, we carefully designed novel algorithms to execute the new operator in a fast and scalable manner;

3. Semantic Validation - we analyzed remote sensing images to support agriculture, following our motivational query with cities and crops, and we also used genetic data to help out in animal breeding. The results of these case studies corroborate the practical usability of the similarity-aware division operator in real applications of high impact. Additionally, we detailed describe how to take advantage of our new operator in digital libraries and in industry.

To support the aforementioned work, we also studied well-known strategies used to implement the traditional division in Relational Database Management Systems (RDBMS), i.e., distinct SQL expressions that are commonly used to represent the division, and propose a new algorithm that is potentially faster than these approaches. 


\subsection{Conclusion}

The rest of the monograph follows a traditional organization: background concepts (Chapter 2), related work (Chapters 3 and 4), proposed operator and algorithms (Chapter 5), experiments (Chapter 6), discussion (Chapter 7) and conclusion (Chapter 8). 



\section{BACKGROUND CONCEPTS}

In this chapter, we present the fundamental concepts related to this MSc work. The chapter begins with a review on the Relational Algebra followed by a discussion of the traditional Relational Division. We also present a brief review about remote sensing images and genetic data - the two types of complex data used in our case studies.

\subsection{Relational Algebra}

The Relational Algebra (CODD, 1972) defines a collection of operators used to manipulate relations of data elements, allowing them to be queried appropriately for each application, e.g., Projection $(\pi)$, Union $(\cup)$ and Join $(\bowtie)$. Every relational operator is defined over one or two relations, and its result is always a relation, which can be used in further operations. The operators are classified as: (a) independent operator, also known as primitives, representing the operations that cannot be expressed by any combination of other operators, and; (b) dependent operators, representing the operators that simplify a sequence of primitive expressions in one single operation (GARCIA-MOLINA; ULLMAN; WIDOM, 2002). For instance, the Intersection $(\cap)$ operator is considered dependent, since it can be rewritten using the Difference (-) operator, as we show in the Expression 2.1, where $T_{1}$ and $T_{2}$ union-compatible relations.

$$
\mathrm{T}_{1} \cap \mathrm{T}_{2} \Leftrightarrow \mathrm{T}_{1}-\left(\mathrm{T}_{1}-\mathrm{T}_{2}\right)
$$

\subsection{Relational Division}

In Relational Algebra (CODD, 1972), the Division $(\div)$ allows simple and intuitive representations for queries with the concept of "for all" (CODD, 1972; CODD, 1990). In fact, it is the only algebraic operator that directly corresponds to the Universal Quantification $(\forall)$ from the Relational Calculus (CODD, 1990). The relational division is similar to the arithmetic operator of division for integer numbers. As well as the arithmetic operator, the relational division 
involves a dividend, a divisor, a quotient and a remainder, known as the operands of the division. In integer arithmetic these operands are always integer values. For example, let us consider the expression $21 \div 5=4$. In this case, 21 is the dividend, 5 is the divisor, 4 is the quotient, and the remainder is 1 . The quotient is 4 since it is the largest integer that, multiplied by the divisor, leads to a value smaller than or equal to the dividend, i.e., $4 * 5 \leq 21$, while the remainder 1 is the difference between the dividend and the result of the aforementioned multiplication, i.e., $21-4 * 5=1$. The relational division works in a similar way, but its operands are relations. Note that these relations do not necessarily contain numeric values, and even when they do, the values may not play a crucial role in the operation.

The relational division is expressed by $\mathrm{T}_{1}\left[\mathrm{~L}_{1} \div \mathrm{L}_{2}\right] \mathrm{T}_{2}=\mathrm{T}_{R}$. In the equation, $\mathrm{T}_{1}, \mathrm{~T}_{2}$ and $\mathrm{T}_{R}$ are the relations that refer to the dividend, the divisor and the quotient, respectively. $L_{1}$ and $L_{2}$ are lists of attributes from $T_{1}$ and $T_{2}$, in that order. Both lists must have the same number of attributes, and each attribute in $L_{1}$ must be union-compatible with its counterpart in $L_{2}$. The quotient relation $T_{R}$ has all the attributes of $T_{1}$ except for those ones listed in $L_{1}$. That is, the schema of $T_{R}$ is given by the relative complement $\overline{L_{1}}$ of list $L_{1}$ with respect to the schema of $\mathrm{T}_{1}$, i.e., $\operatorname{Sch}\left(\mathrm{T}_{R}\right)=\overline{\mathrm{L}_{1}}=\operatorname{Sch}\left(\mathrm{T}_{1}\right)-\mathrm{L}_{1}$. For example, let us consider the division $T_{1}\left[\left(A_{5}, A_{4}, A_{2}\right) \div\left(A_{7}, A_{6}, A_{8}\right)\right] T_{2}=T_{R}$ to be executed over relations $T_{1}$ and $T_{2}$. Assume that $T_{1}$ is defined by a schema $\operatorname{Sch}\left(T_{1}\right)=\left(A_{1}, A_{2}, A_{3}, A_{4}, A_{5}\right)$, in which $A_{1}, A_{2}, A_{3}, A_{4}$ and $A_{5}$ are attributes. $T_{2}$ has a schema $\operatorname{Sch}\left(T_{2}\right)=\left(A_{6}, A_{7}, A_{8}\right)$ with attributes $A_{6}, A_{7}$ and $A_{8}$. In this setting, it is required that attribute list $L_{1}=\left(A_{5}, A_{4}, A_{2}\right)$ has the same number of attributes of list $L_{2}=\left(A_{7}, A_{6}, A_{8}\right)$, which is true, and every pair of attributes $\left[A_{5}, A_{7}\right],\left[A_{4}, A_{6}\right]$ and $\left[A_{2}, A_{8}\right]$ must share the same data domain. The schema of the quotient relation $T_{R}$ is $\operatorname{Sch}\left(\mathrm{T}_{R}\right)=\left(\mathrm{A}_{1}, \mathrm{~A}_{3}\right)$.

Remember that the quotient in the arithmetic integer division is the largest integer that, multiplied by the divisor, defines a value smaller than or equal to the dividend, i.e., quotient $*$ divisor $\leq$ dividend. The remainder is the difference between the dividend and the result of multiplying the quotient by the divisor, i.e., dividend - quotient $*$ divisor. The relational division is defined in a very similar manner: the quotient relation $T_{R}$ is the subset of $\pi_{\left(\overline{L_{1}}\right)}\left(\mathrm{T}_{1}\right)$ with the largest possible cardinality, such that $\mathrm{T}_{R} \times \mathrm{T}_{2} \subseteq \mathrm{T}_{1}$, and the remainder relation is given by $\mathrm{T}_{1}-\mathrm{T}_{R} \times \mathrm{T}_{2}$. As a consequence, it is commonly said (CODD, 1972) that in the relational division the operators Cartesian Product $(\times)$ and Difference $(-)$ play the role of the arithmetic operators Multiplication $(*)$ and Difference $(-)$ from the division of integer numbers. The relation of inclusion $\subseteq$ refers to the inequality $\leq$.

Note that the relational division can be alternatively expressed by a combination of other relational operators, specifically, the Cartesian Product $(\times)$, Difference $(-)$ and Projection $(\pi)$. Equation 2.2 defines this equivalence.

$$
\mathrm{T}_{1}\left[\mathrm{~L}_{1} \div \mathrm{L}_{2}\right] \mathrm{T}_{2} \equiv \pi_{\left(\overline{\mathrm{L}_{1}}\right)}\left(\mathrm{T}_{1}\right)-\pi_{\left(\overline{\mathrm{L}_{1}}\right)}\left(\left(\pi_{\left(\overline{\mathrm{L}_{1}}\right)}\left(\mathrm{T}_{1}\right) \times \pi_{\left(\mathrm{L}_{2}\right)}\left(\mathrm{T}_{2}\right)\right)-\mathrm{T}_{1}\right)
$$

Although being possibly represented by other relational operators, the division plays a 
significant role in the Relational Algebra. The main reasons are: (a) the division allows simple representations for queries with the concept of "for all" - we emphasize the considerable complexity of its equivalent representation in Equation 2.2; (b) the division is the only operator that directly corresponds to the Universal Quantification $(\forall)$ from the Relational Calculus (CODD, 1990); (c) it improves and makes intuitive the understanding of complex queries, and, fundamentally; (d) it supports the creation of efficient algorithms to execute the queries. Note that an equivalent situation occurs with the operator Join $(\bowtie)$, which, besides having alternative representations with other operators, it also makes it easier to write and to understand queries. Especially, it supports the development of a number of efficient algorithms, focusing either on specific cases of join or on its generic instance, which are largely used in RDBMS.

\subsection{Remote Sensing Images}

Remote sensing is the technology that allows obtaining images and other types of data from the terrestrial surface. This technique uses sensors to gather and record from a distance, without direct contact, the reflected or absorbed energy of the ground (FITZ, 2008). The interaction of the electromagnetic energy and the objects on the terrestrial surface is the source of the remote sensing data. These interactions give the physicochemical and biological properties of these objects, which the images and other remote sensing data capture. The amount of reflected or absorbed energy varies with the kind of the object and is given in several lengths of waves. Each length of the wave is captured by the sensor in a different spectral band, as we illustrate in Figure 2.1. Several applications use this kind of data. In agriculture, for example,

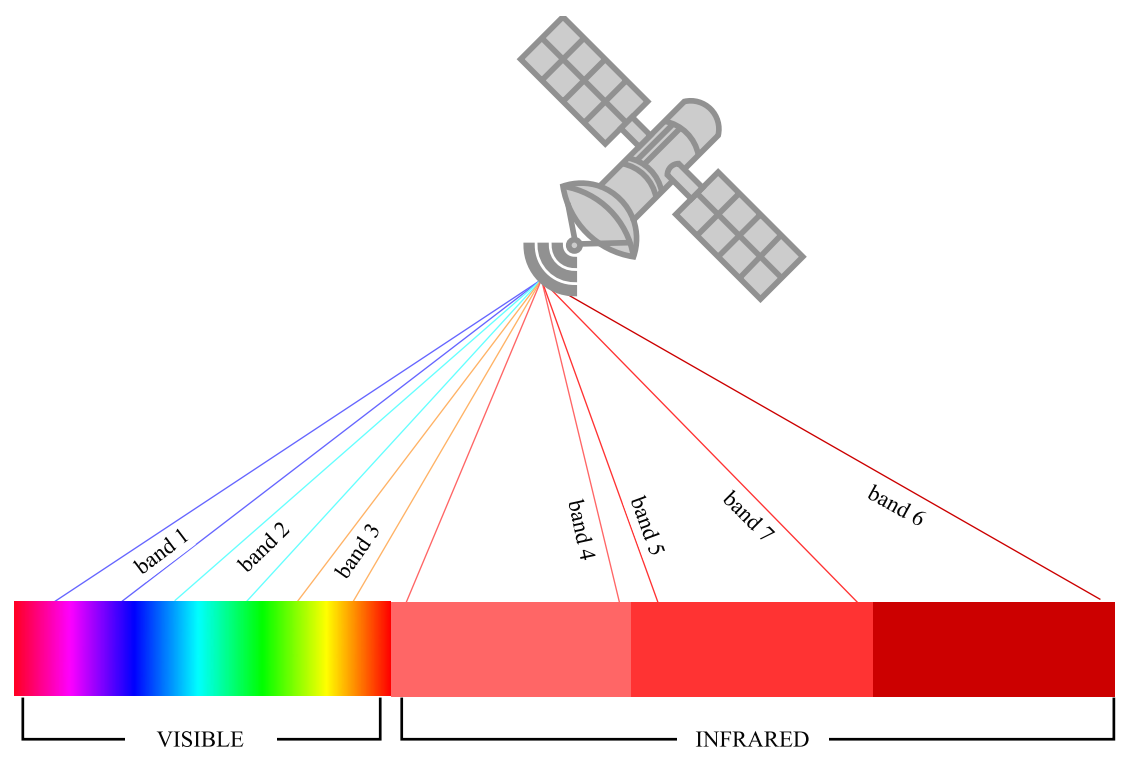

Figure 2.1 - Example of the spectrum range of a remote sensing satellite.

this is a powerful tool to support crop analysis. It can help to distinguish small changes in the field such as the differences between a healthy and an unhealthy vegetation, which appear very differently in the spectral bands that are not visible by humans. Infrared bands are also very useful to identify different varieties, like bare soil, ground crops, and trees, for example. 


\subsection{Genetic Data}

The genome of an individual is the complete set of genetic information in its organism. It is associated to all phenotypic expressions of the individual, varying from its predisposition to develop diseases until the color of its eyes, for example (ZHANG et al., 2004). The genome is stored in molecules of DNA (deoxyribonucleic acid) called chromosomes. Each chromosome carries the genes responsible to develop the organism traits, formed from a chain of four nucleotide bases - A, T, C, and G. Population of the same species has almost all the DNA carrying the same nucleotides at a specific position in the DNA sequence, however, if more than one percent of the population have variations at a specific position, the position is therefore classified as a Single Nucleotide Polymorphism, or SNP (pronunced "snip"), as it is illustrated in Figure 2.2. When a SNP occurs within a gene, then it is described as having more than one allele, that is, a pair of genes that appear at a particular location on a particular chromosome and control the same characteristics. For example, it is estimated that there are over 10 million SNP markers in the human genome and that at least 300 thousand of them involve significant genetic variation. Datasets containing SNPs are usually represented in RDBMS by positions at chromosomes and the corresponding pair of alleles, each one expressed by the numbers 1 or 2 . Table 6.1 illustrates a toy dataset with 9 SNPs referring to 3 individuals. Note that it is unfeasible to represent SNPs with attribute columns, since each individual commonly have tens of thousands SNPs.

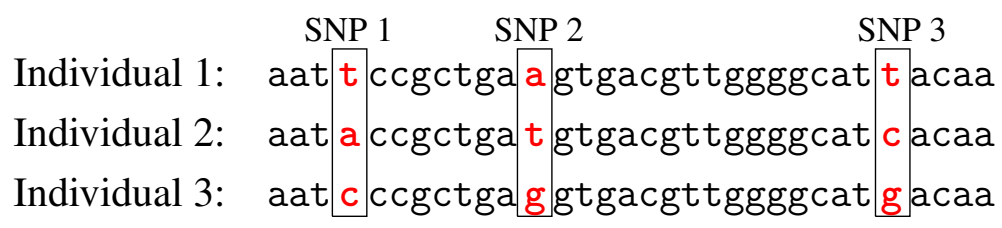

Figure 2.2 - An example of SNPs along chromosomes of individuals.

Table 2.1 - Toy dataset containing genetic data of 3 individuals.

\begin{tabular}{|c|c|c|c|}
\hline ID & Position & Allele 1 & Allele 2 \\
\hline 1 & 0.10 & 1 & 2 \\
1 & 44.5 & 2 & 2 \\
1 & 84.0 & 2 & 1 \\
2 & 0.10 & 2 & 2 \\
2 & 44.5 & 1 & 1 \\
2 & 84.0 & 1 & 2 \\
3 & 0.10 & 1 & 2 \\
3 & 44.5 & 2 & 1 \\
3 & 84.0 & 2 & 2 \\
\hline
\end{tabular}




\subsection{Conclusion}

This chapter introduced background concepts that are used as a basis for this MSc work. The next chapter presents related works regarding existing implementations for the traditional operator of division, as well as works focused on how to support similarity comparison in other relational operators. 

This chapter comments on related works. We first focus on existing implementations of the traditional operator of division. Then, we describe efforts already made to support similarity comparison in other relational operators.

\subsection{Relational Division}

Several works investigate the relational division. In (LEINDERS; BUSSCHE, 2005), Leinders and Van den Bussche demonstrate that any implementation using only primitive operators of the Relational Algebra, e.g., Equation 2.2 from Chapter 2, has a minimum time complexity $\mathrm{O}\left(n^{2}\right)$, in which $n$ is the cardinality of the dividend relation. Fortunately, the authors also describe alternative approaches with counting and sorting-based strategies being used to optimize the execution up to a time complexity $\mathrm{O}(n \log n)$.

The work in (MATOS; GRASSER, 2001) investigates possible implementations for the division in Structured Query Language (SQL), and reports results of a number of experiments performed to compare them. Although being more than ten years old and using limited volume and diversity of data in the experiments, this work indicates that the counting-based approach is better than the other ones studied, since it is faster and minimizes disk accesses. Similar results were also reported in the undergraduate thesis of the MSc candidate (GONZAGA; CORDEIRO, 2016), in which six distinct SQL implementations for the division were compared using datasets with volume and diversity substantially larger than those of (MATOS; GRASSER, 2001).

Celko (CELKO, 2009) also investigated diverse implementations of the division in SQL, and discussed a number of potential applications for it. The work covers alternative divisionbased queries that slightly differ from the operator originally introduced by Codd (CODD, 1972), including the concepts of division without the remainder and division with multiple divisors. However, no experiment was performed to validate these custom queries nor to evaluate their efficiency. In another work (CAMPS, 2014), Camps improved Celko's study by reporting 
qualitative and quantitative results for the division implemented in SQL, considering both its original definition and some of its variants, like the division with multiple divisors. The main contribution of the work is to identify optimization strategies by means of filtering out irrelevant data before and during the execution of the queries. Experiments indicate that the proposed algorithms are efficient, but they work only on special cases of the division, heavily focused on one single application. Despite the many qualities present in the works of Celko (CELKO, 2009) and Camps (CAMPS, 2014), it is important to note that none of the alternative division-based queries studied in these works is well suited to process data of a more complex nature, such as the remote sensing images in our motivational example with cities and crops (see Figures 1.1d, 1.1e and 1.1f) from Chapter 1.1.

Another study branch present in the literature that discusses the extension of the Relational Division operator, is the set of works that approach it using fuzzy logic (GALINDO; URRUTIA; PIATTINI, 2005). The work (TAMANI; LIETARD; ROCACHER, 2013) explores the inclusion of fuzzy bipolar conditions to the relational division, which is the ability to express queries with a mandatory condition $c$ and an optional wish $w$, meaning "satisfy $c$ and if possible satisfy $w$ ". Its main idea consists in a tolerant relational division, which selects almost all elements of the dividend that are related to the divisor. The work (GALINDO; MEDINA; GARRIDO, 2001) uses the generalized model of fuzzy relational database (MEDINA; PONS; MIRANDA, 1994), which specifies a different data structure and a modified Relational Algebra to handle imprecise information. The comparisons of the relational division operator are relaxed by setting a threshold on the possibility distributions of the attributes values. The work (BOSC; PIVERT, 1999) also proposes a new semantics to the division operator, aiming fuzzy relations and the notion of ideal values, which extend the concept of threshold weights and grades of importance for the tuples when performing the division in fuzzy relations. The common idea of all these works is that they aim to extend the relational division semantics to a fuzzy domain, which implies having fuzzy relations with vague values using linguistic labels, weighted tuples and grades with different meanings for the attributes. None is concerned in extending the relational division operator to handle nonidentity comparisons in complex data, which is different from imprecise and uncertain data, using similarity measures inside the context of

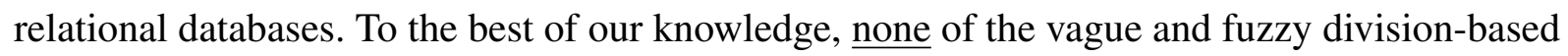
operators present in literature is well suited, nor has ever been used, to process data of a more complex nature - complex, not imprecise/uncertain - such as the remote sensing images in our motivational example with cities and crops (see Figures 1d, 1e and 1f), genetic data, large text, video collections, fingerprints, large graphs extracted from social networks, and many other types of complex objects that must be analyzed by modern applications. 


\subsection{Similarity-awareness in the Relational Algebra}

Several works aim at supporting similarity comparison in operators of the Relational Algebra. The vast majority of them focuses on the Selection (SILVA et al., 2010; SILVA et al., 2013; BARIONI et al., 2009; SANTOS et al., 2013; BUDÍKOVÁ; BATKO; ZEZULA, 2012; BELOHLAVEK; VYCHODIL, 2010), in which similarity awareness is achieved by means of range queries, nearest neighbors queries, and their many variants. The range query selects all tuples that are "similar enough" to another tuple chosen by the user to be the query center, while the nearest neighbors query returns the $k$ tuples most similar to the center. In both approaches, similarity is measured by a given distance function. The user must also specify a distance threshold in range queries, and a value for $k$ in nearest neighbors queries.

Existing proposals for the similarity-aware Join (SILVA et al., 2015; JACOX; SAMET, 2008; SILVA; PEARSON, 2012; SILVA et al., 2013; KALASHNIKOV, 2013; SILVA; AREF; ALI, 2010) also follow the aforementioned principles, usually considering range and nearest neighbors queries associated with a given distance function. The main approaches are: (1) Range Distance Join that retrieves every pair of tuples with distance up to a given threshold; (2) kNN Join that, for each tuple of the left relation, retrieves its $k$ most similar tuples from the right relation, and; (3) k-Distance Join that retrieves the overall $k$ most similar pairs of tuples.

Clustering/Grouping data by similarity is a well-known task in a variety of research areas, such as: data mining, databases, statistics and biology (CORDEIRO; FALOUTSOS; Traina Jr., 2013; CORDEIRO et al., 2013; LI; GODZIK, 2006; MAULIK; BANDYOPADHYAY; MUKHOPADHYAY, 2011). Nevertheless, very few works focus on similarity-aware Grouping and Aggregation in the context of the Relational Algebra. One exception is the work of Silva et al. (TANG et al., 2016a; TANG et al., 2016b; SILVA; AREF; ALI, 2009). Unfortunately, a number of open questions remain, like: (1) how to identify appropriate execution plans for queries with similarity-aware grouping operations together with the existing relational operations; (2) how to define the new operations algebraically, and; (3) how to develop fast and scalable algorithms to execute them.

The relational set-based operators have also been studied. Pola et al. (POLA et al., 2015; POLA et al., 2013) introduced the SimSets, which are sets of data without any pair of similar elements with regard to a given distance function and a similarity threshold. The function must be a metric, thus the usual absence of identical elements is maintained. The authors present efficient algorithms to extract a SimSet from any dataset, and to perform set-based operations on SimSets, like Union $(\cup)$, Intersection $(\cap)$ and Difference $(-)$. Similarity-aware set operators have also been proposed by Al Marri et al. (MARRI et al., 2014; MARRI et al., 2016). The authors present efficient query processing algorithms to evaluate the new operators, and successfully implements them inside an open-source database system, namely PostgreSQL.

This section discussed some of the many efforts already made to develop a similarity- 
aware $R D B M S$, especially interested in works that extend operators of the Relational Algebra. To the best of our knowledge, no one has ever focused on the Division, despite the fact that this operator is clearly unsuitable to process complex data. Section 1.2 from Chapter 1.1 and Section 5.1 from Chapter 5 describe its main limitations. In this MSc work, we introduce a new branch of research by presenting the first similarity-aware division database operator.

\subsection{Conclusion}

This chapter commented on related works. We discussed works that deal with the traditional relational division, including studies performed about its many possible implementations, and their corresponding runtime complexities. We also covered works that deal with the inclusion of similarity-awareness in relational operators, emphasizing that none of them addresses the relational division. The next chapter presents an initial work that we have developed to better understand the traditional division, which we used as a basis to perform our main work on the similarity-aware version of this operator. 


\section{RELATIONAL DIVISION}

This chapter presents a preliminary work that we have performed to better understand the traditional relational division. To achieve this goal, we designed a novel data generator that supports the analysis of division queries, and used it to compare two well-known SQL implementations for the division in datasets with distinct data volume and diversity. Also, we carefully designed a novel procedural algorithm for the division, and implemented it in a stored procedure as an initial prototype. Experiments show that the new algorithm is as fast as the counting-based SQL approach - the state-of-the-art implementation - in spite of the intrinsic overhead of switching contexts from SQL and the procedural language. These results suggest that our algorithm may be useful as an efficient physical operator to be implemented in the core of the RDBMS.

\subsection{SQL Implementations for the Division}

This section presents the two SQL implementations that we studied. To support the discussion, we consider a generic division query expressed as $T_{1}\left[L_{1} \div L_{2}\right] T_{2}=T_{R}$. In the equation, $T_{1}, T_{2}$ and $T_{R}$ are relations that refer to the dividend, the divisor and the quotient, respectively. $L_{1}$ and $L_{2}$ are lists of attributes from $T_{1}$ and $T_{2}$, in that order. There exists a number of SQL expressions that implement the relational division. Based on the literature review presented in the previous chapter, we decided to evaluate two well known approaches: Query 4.1, that strictly follows the division representation using primitive operators of the Relational Algebra - Equation 2.2 from Chapter 2, and; Query 4.2, that uses tuple counting to speed-up the execution, being considered the state-of-the-art implementation by the literature. 


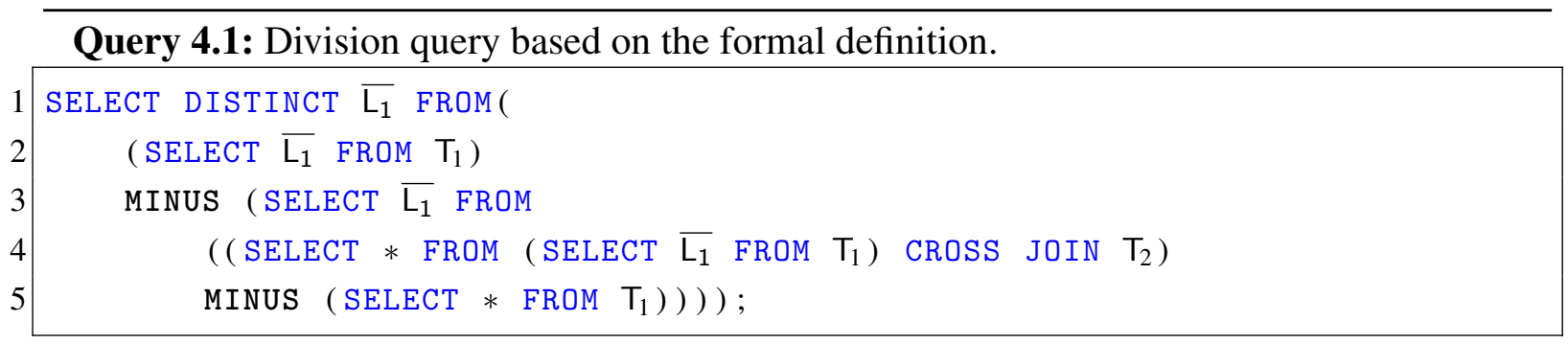

Query 4.2: Division query based on counting tuples.

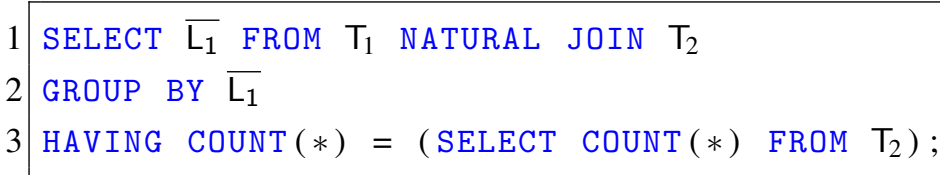

\subsection{Proposed Algorithms}

This section presents two algorithms that we designed in this study: (a) one new procedural algorithm to execute the traditional division, and; (b) a novel data generator well suited to create synthetic data with varying volume and diversity for the operator.

\subsubsection{Index-Division}

We developed the new algorithm Algorithm 1 for the division operation, takingas premise the existence of index structures over the dividend attributes in list $L_{1}$. It assumes that all candidates in $T_{1}$ satisfy the requirements of $T_{2}$ at the beginning, by initializing relation $T_{R}$ in Line 2. Then, for each requirement in $T_{2}$, it is performed an indexed query in relation $T_{1}$, using the requirement as the query center. It recovers then for each tuple retrieved by the query, to which candidate it belongs to. Then, the valid candidates in $T_{R}$ are updated with the intersection of the last iteration's valid groups and the candidates that meet the current requirement. Therefore, at the end of the algorithm, only candidates that meet all requirements will be in $\mathrm{T}_{R}$.

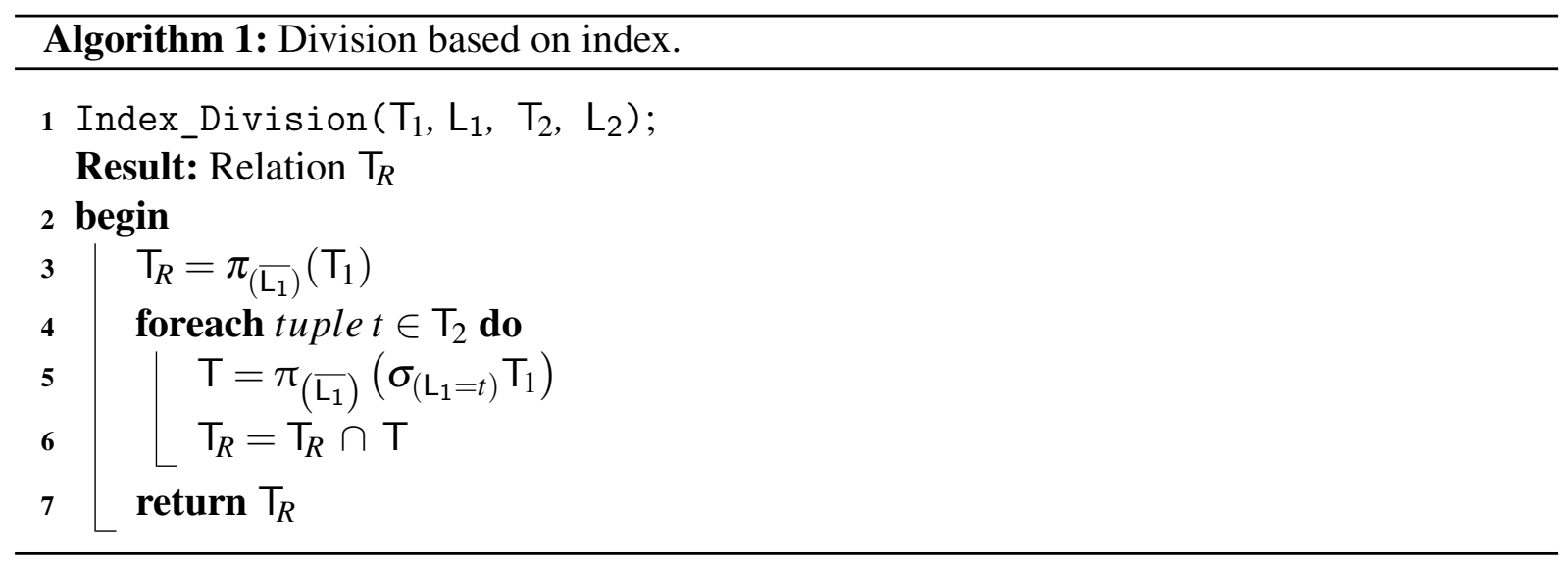

The proposed algorithm performs, for each tuple in relation $T_{2}$ an indexed search query in relation $T_{1}$. State-of-the-art index structure s like the B-tree and its variants perform search 
queries with a complexity of $\mathrm{O}(\log n)$, where $n$ is the relation cardinality. Thus, the theoretic runtime complexity of our algorithm is $\mathrm{O}(m \log n)$, where $n$ and $m$ are the number of tuples in relations $T_{1}$ and $T_{2}$, respectively. The current version of our algorithm is implemented by stored procedures with PL/SQL in the Oracle database system.

\subsubsection{Division Data Generator}

To study the algorithms performance in different scenarios, we developed a data generator using the following parameters: (1) Cardinality, the number of tuples in the dividend $T_{1}$ and in the divisor $T_{2}$; (2) Number of candidates, the cardinality of $\pi_{\left(\overline{L_{1}}\right)}\left(T_{1}\right)$ representing the number of candidates to be evaluated in the operation; (3) Correlation, the percentage of candidates, from the total, that satisfy all the requirements in $\mathrm{T}_{2}$ thus being part of the result, e.g, 10\% of correlation means that only $10 \%$ of all candidates will be selected in the final result; (4) Variability, the differences in size between candidates, i.e., groups of tuples from $T_{1}$ that share the same value for attributes $L_{1}$, adjusting the number of tuples in each group. High variability means that some candidates have many tuples, and others have just a few. Low variability means that almost all candidates have nearly the same number of tuples;

All these parameters are linked and affect one another, thus, our data generator works as follows: (1) set the cardinality of the divisor relation $\mathrm{T}_{2}$; (2) set the number of candidates based on the correlation value; (3) create the group of tuples in $T_{1}$ for each candidate, taking the variability value into account; (4) define attribute values for each one of these tuples ensuring that only the candidates that are part of the result, satisfy all the tuples in the divisor, according to the correlation value defined in the previous step.

\subsection{Experiments}

We performed experiments to compare our proposed algorithm with the well known SQL implementations in Query 4.1 and 4.2. All the experiments were performed in a machine with an Intel i7 2.67GHz processor and 12Gb RAM, using the Oracle Database 11g Express running on Ubuntu 14. All the queries used index structures to optimize their performance.

\subsubsection{Synthetic Data}

We performed experiments varying the parameters of our data generator. Each plot in Figure 6.4 represents the variation of a single parameter while the others are fixed in: (1) dividend size, one million of tuples; divisor size, ten tuples; (2) one hundred of candidates; (3) $10 \%$ of correlation; (4) $50 \%$ of variability; The plots in Figures $4.1 \mathrm{a}$ and $4.1 \mathrm{~b}$ show that the cardinalities of the dividend and divisor are the only aspects that have significant impact on the query runtime. The plots referring to the number of candidates, correlation and variability - see Figures 4.1c, 4.1d and 4.1e - present no significant variations in runtime. The plot presented in Figure 4.1g, 
where we studied 40 test cases varying parameters as:(1) dividend, from hundred to million of tuples; divisor, from one tuple to one hundred of tuples; (2) from two candidates to hundreds; (3) from $1 \%$ to $100 \%$ of correlation; and the variability fixed in 50\%; shows that, overall, although the execution plan for the counting-based query - see Execution Plan 4.1 - is different from our IndexDivision algorithm, both approaches have almost the same performance. The query that translates the formal definition have the worst performance for all test cases. Theses results can be associated to the plot in Figure 4.1f, which shows the average processing cost reported by the Oracle DBMS for all queries.

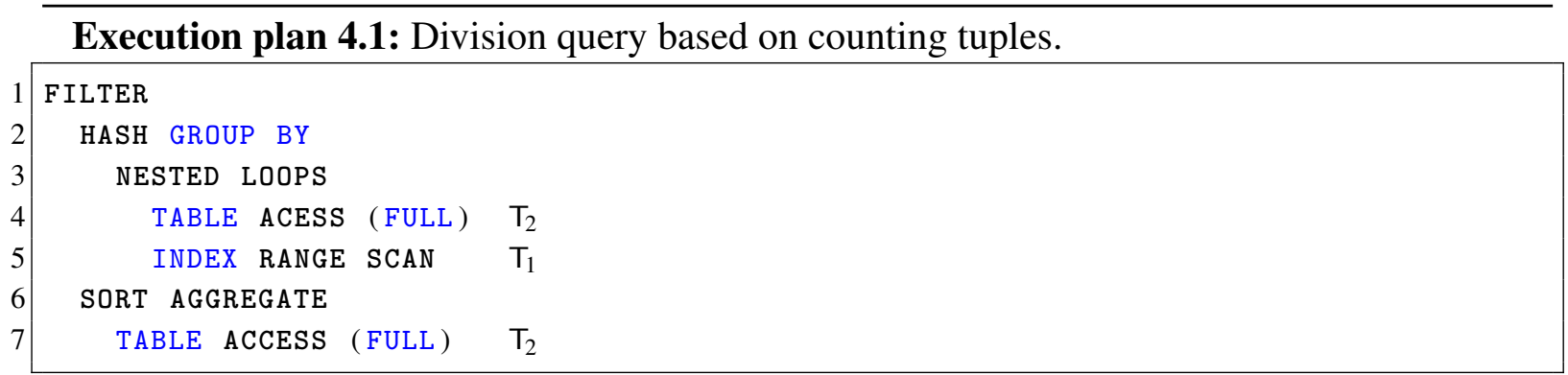

\subsection{Conclusion}

In this chapter we presented a novel data generator and evaluated two well known SQL implementations for the traditional relational division, showing that the cardinality of the input relations is the characteristic that most affects the execution runtime. Also, we presented the novel IndexDivision algorithm, which had barelly the same performance compared with the best division query in SQL presented in the literature, being slightly faster overall. Note that our IndexDivision algorithm was implemented in stored procedures using PL/SQL, which means that the algorithm has the bottleneck of switching contexts from SQL and the procedural language. Thus, we believe that an implementation of the IndexDivision inside the core of the DBMS could achieve the best performance overall.

The next chapter presents the main contribution of this MSc work - the new Similarityaware relational division database operator. 


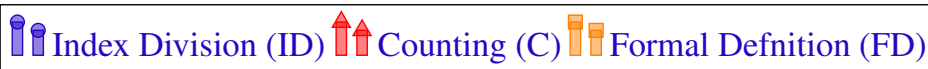
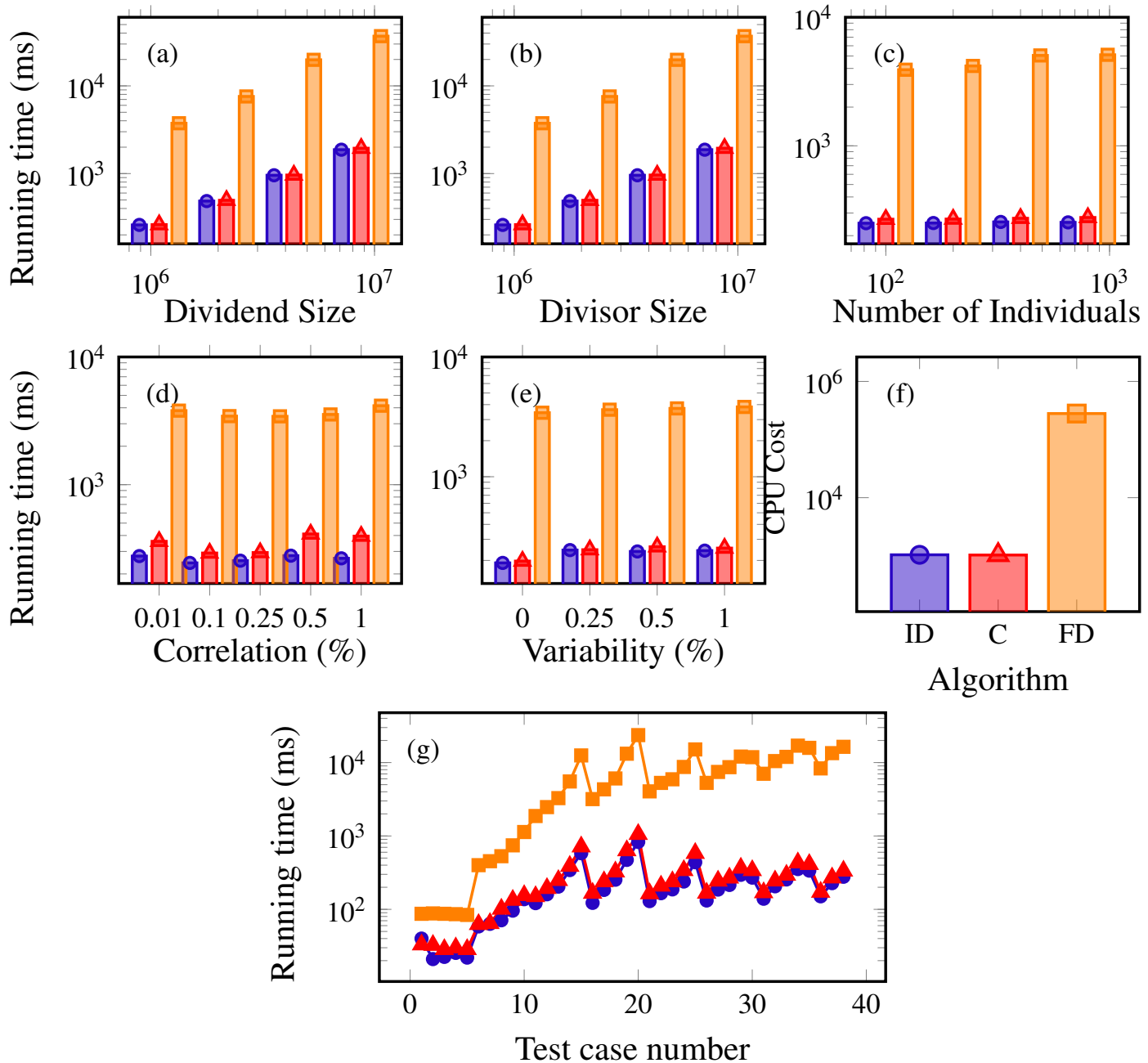

Figure 4.1 - Relational Division queries performance on different use cases. 



\section{SIMILARITY-AWARE DIVISION}

This chapter presents the main contribution of this MSc work: the Similarity-aware relational division operator. We first present the operator, including formal definitions for it. Then we propose two novel algorithms to execute the similarity-aware division in a fast and scalable manner.

\subsection{Proposed Similarity-aware Division Operator}

This section presents the new Similarity-aware Division $(\hat{\dot{\div}})$ database operator. To make it possible, we first investigate attribute comparisons that are intrinsically performed by the traditional operator of division. The results obtained are then used to present our proposal.

\subsubsection{Intra and Inter-relation Comparisons}

The relational division includes intrinsic comparisons of attribute values that, by the very definition, are always based on identity $(=)$. Remember that the quotient relation $\mathrm{T}_{R}$ in an operation of division $T_{1}\left[L_{1} \div L_{2}\right] T_{2}=T_{R}$ is the subset of $\pi_{\left(\overline{L_{1}}\right)}\left(T_{1}\right)$ with the largest possible cardinality, provided that $\mathrm{T}_{R} \times \mathrm{T}_{2} \subseteq \mathrm{T}_{1}$. It is interesting to note that this definition partitions relation $T_{1}$ into $\mathrm{K} \geq 0$ distinct groups of tuples, in such a way that each group $\mathrm{T}_{G_{k}} \subseteq \mathrm{T}_{1}$ represents one candidate tuple for $T_{R}$, which is given by $\pi_{\left(\overline{L_{1}}\right)}\left(T_{G_{k}}\right)$. The following conditions apply:

$$
\begin{gathered}
\mathrm{T}_{1}=\bigcup_{k=1}^{\mathrm{K}} \mathrm{T}_{G_{k}} \text {, and } \\
\forall \mathrm{T}_{G_{k}}, \mathrm{~T}_{G_{k^{\prime}}} \subseteq \mathrm{T}_{1}:\left(\pi_{\left(\overline{\mathrm{L}_{1}}\right)}\left(\mathrm{T}_{G_{k}}\right) \cap \pi_{\left(\overline{\mathrm{L}_{1}}\right)}\left(\mathrm{T}_{G_{k^{\prime}}}\right)=\varnothing\right) \mid k \neq k^{\prime} .
\end{gathered}
$$

Relation $T_{1}$ is partitioned according to its active domain, so that the tuples of each group $\mathrm{T}_{G_{k}}$ must share the same values for the attributes in $\overline{\mathrm{L}_{1}}$. That is:

$$
\forall \mathrm{T}_{G_{k}} \subseteq \mathrm{T}_{1}:\left(\forall t_{i}, t_{i^{\prime}} \in \mathrm{T}_{G_{k}}:\left(\forall \mathrm{A}_{\mathrm{j}} \in \overline{\mathrm{L}_{1}}:\left(t_{i}\left[\mathrm{~A}_{\mathrm{j}}\right]=t_{i^{\prime}}\left[\mathrm{A}_{\mathrm{j}}\right]\right)\right)\right),
$$


in which $t_{i}\left[\mathrm{~A}_{\mathrm{j}}\right]$ and $t_{i^{\prime}}\left[\mathrm{A}_{\mathrm{j}}\right]$ are respectively the values of an attribute $\mathrm{A}_{\mathrm{j}}$ for tuples $t_{i} \in \mathrm{T}_{G_{k}}$ and $t_{i^{\prime}} \in \mathrm{T}_{G_{k}}$. Note that the values are always compared by identity, i.e., $t_{i}\left[\mathrm{~A}_{\mathrm{j}}\right]=t_{i^{\prime}}\left[\mathrm{A}_{\mathrm{j}}\right]$.

We name these comparisons as intra-relation, since they process distinct tuples of the dividend $\mathrm{T}_{1}$. To illustrate the intra-relation comparisons, let us consider again our motivational example with cities and crops from Figures 1.1, which was originally discussed in Chapter 1.1. Note that we replicate the same example in Figure 5.1, for the reader's convenience. The query in Figures 5.1a, 5.1b and 5.1c identifies cities well-suited to produce a particular type of crop based on textual tags that describe city regions and crop requirements. Formally, it is defined in Equation 5.1.

$$
\text { CityRegions }[(\text { Region }) \div(\text { Region })] \text { Requirements }=\text { Cities }
$$

Relations CityRegions, Requirements and Cities are the dividend, the divisor and the quotient, respectively. Note that CityRegions is implicitly partitioned into three distinct groups of tuples, $\mathrm{T}_{G_{1}}, \mathrm{~T}_{G_{2}}$ and $\mathrm{T}_{G_{3}}$, which we highlight with distinct shades of gray in Figure 5.1a. The partition is based on the active domain of the attributes in $\overline{\mathrm{L}_{1}}=\overline{(\text { Region })}=($ City). The groups are therefore defined by the values of a single attribute in this specific example: attribute City with active domain \{Campinas, São Carlos, Araraquara\}. All tuples of each group $\mathrm{T}_{G_{k}} \subseteq$ CityRegions must share the same value in this attribute, so that the projection $\pi_{(\text {City })}\left(T_{G_{k}}\right)$ identifies a single candidate tuple for the quotient relation Cities, that is, a candidate city for the crop production. Groups $\mathrm{T}_{G_{1}}, \mathrm{~T}_{G_{2}}$ e $\mathrm{T}_{G_{3}}$ respectively represent tuples 〈Campinas〉, 〈São Carlos〉 and 〈Araraquara〉.

It is interesting to note that, besides comparing attribute values in distinct tuples of relation $\mathrm{T}_{1}$ via intra-relation comparisons, the division also includes inter-relation comparisons, and again, they are always performed by identity (=). Specifically, attribute values from the dividend $T_{1}$ are compared with their counterparts in the divisor $T_{2}$, according to attribute lists $L_{1}$ e $L_{2}$. Remember that the quotient $T_{R}$ of a division query $T_{1}\left[L_{1} \div L_{2}\right] T_{2}=T_{R}$ is the subset of $\pi_{\left(\overline{L_{1}}\right)}\left(\mathrm{T}_{1}\right)$ with the largest possible cardinality, provided that $\mathrm{T}_{R} \times \mathrm{T}_{2} \subseteq \mathrm{T}_{1}$, and the dividend $\mathrm{T}_{1}$ is implicitly partitioned into $\mathrm{k} \geq 0$ non-overlapping groups of tuples, each group $\mathrm{T}_{G_{k}} \subseteq \mathrm{T}_{1}$ identifying a candidate tuple for $T_{R}$ that is given by $\pi_{\left(\overline{L_{1}}\right)}\left(T_{G_{k}}\right)$. In this setting, the candidate tuple of a group $T_{G_{k}}$ belongs to $T_{R}$ if and only if the equijoin $T_{G_{k}} \stackrel{\left(L_{1}=L_{2}\right)}{\bowtie} T_{2}$ has a match, i.e., true join predicate, for every tuple of the divisor $T_{2}$. Therefore, it is possible to redefine the quotient relation $\mathrm{T}_{R}$ by Equation 5.2.

$$
\mathrm{T}_{R}=\bigcup_{k=1}^{\kappa} \begin{cases}\pi_{\left(\overline{\mathrm{L}_{1}}\right)}\left(\mathrm{T}_{G_{k}}\right), & \text { if } \pi_{\left(\mathrm{L}_{2}\right)}\left(\mathrm{T}_{G_{k}} \stackrel{\left(\mathrm{L}_{1}=\mathrm{L}_{2}\right)}{\aleph^{\circ}} \mathrm{T}_{2}\right)=\mathrm{T}_{2} \\ \varnothing, & \text { otherwise }\end{cases}
$$

The join predicate is $\mathrm{L}_{1}=\mathrm{L}_{2}$, thus individual attribute values from the dividend $\mathrm{T}_{G_{k}} \subseteq \mathrm{T}_{1}$ are compared by identity with their counterparts in the divisor $T_{2}$. To illustrate this fact, let us 


\begin{tabular}{|c|c|}
\hline City & Region \\
\hline Campinas & Water* \\
\hline Campinas & Urban Area \\
\hline Campinas & Silos \\
\hline Campinas & Road \\
\hline São Carlos & Bare Soil \\
\hline São Carlos & Urban Area \\
\hline São Carlos & Forest \\
\hline São Carlos & Silos \\
\hline São Carlos & Water* \\
\hline Araraquara & Urban Area \\
\hline Araraquara & Bare Soil \\
\hline
\end{tabular}

(a) CityRegions

\section{$\div \quad$\begin{tabular}{|c|}
\hline Region \\
\hline Water $^{*}$ \\
\hline Bare Soil \\
\hline Silos \\
\hline Urban Area \\
\hline São Carlos \\
\hline (c) Cities \\
\end{tabular}}

(b) Requirements

* example of identical $(=)$ region tags

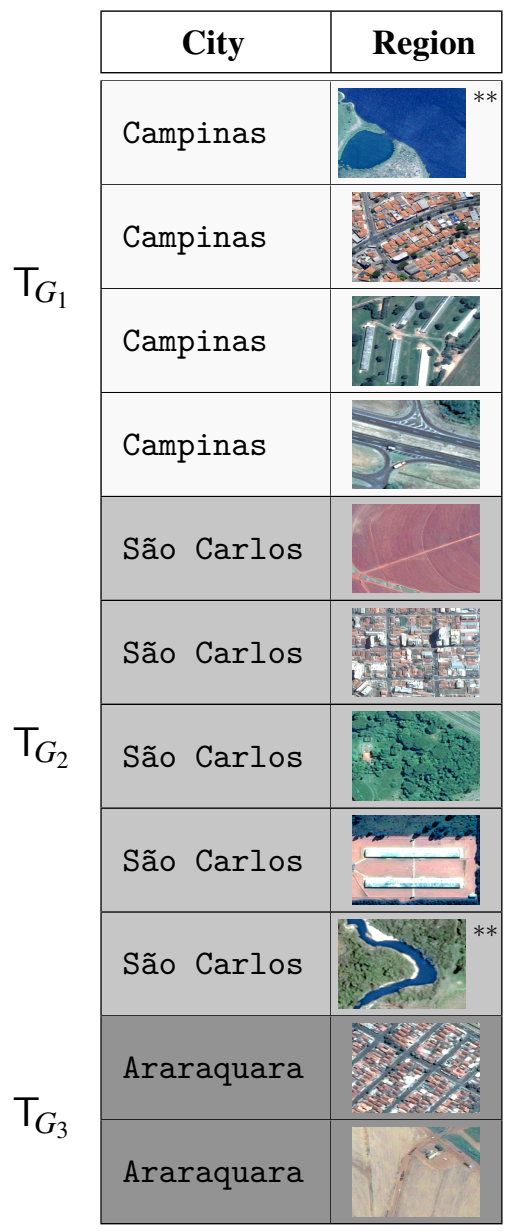

(d) CityRegions

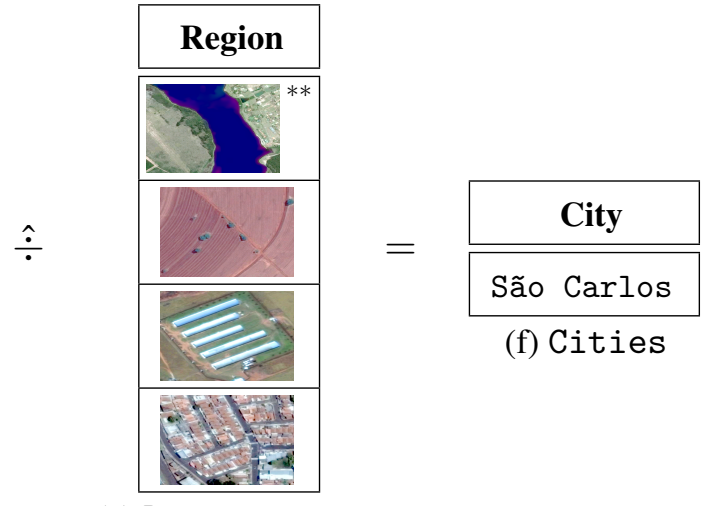

(e) Requirements

** example of similar $(\hat{=})$ tiles of water extracted from remote sensing images

Figure 5.1 - Example of the division used to select cities well suited to produce a particular type of crop. Top: textual tags are compared by identity $(=)$. Bottom: tiles extracted from remote sensing images are compared by similarity $(\hat{=})$. Best viewed in color. 
consider again our running example with cities and crops from Figures 5.1a, 5.1b and 5.1c, defined in Equation 5.1. The query partitions the dividend CityRegions into three groups of tuples, each one identifying a candidate tuple for the quotient Cities. Tuple 〈São Carlos〉 from group $\mathrm{T}_{G_{2}}$ is in Cities because every tuple of Requirements has a match in the equijoin $(($ Region $)=($ Region $))$

$\mathrm{T}_{G_{2}} \bowtie$ Requirements. On the other hand, tuple $\langle$ Campinas $\rangle$ from group $\mathrm{T}_{G_{1}}$ is not in the quotient since there is no match for tuple $\langle$ Bare Soil $\in \in$ Requirements in the corresponding equijoin. Tuple $\left\langle\right.$ Araraquara is also out of relation Cities, as group $\mathrm{T}_{G_{3}}$ has no match for neither the Silos nor the Water requirements.

\subsubsection{Similarity-awareness in the Division}

To develop a similarity-aware operation of division one must extend both intra and inter-relation comparisons. The former must be re-engineered to find candidates for the quotient $\mathrm{T}_{R}$ by grouping together the tuples of the dividend $\mathrm{T}_{1}$ with similar values in the attributes of $\overline{\mathrm{L}_{1}}$, while the latter should populate $\mathrm{T}_{R}$ with the candidates that have at least one tuple similar to each tuple of the divisor $T_{2}$, taking into account only the attributes of $L_{1}$ and $L_{2}$. To support

Query: CityRegions $[($ Region $) \hat{\div}$ (Region) $]$ Requirements $=$ Cities

Schema: $\operatorname{Sch}$ (CityRegions) $=$ (GeoPosition, Region)

- GeoPosition: array of latitude and longitude values.

- Region: satellite image.

$S c h$ (Requirements $)=($ Region $)$

- Region: satellite image.

$\operatorname{Sch}($ Cities $)=($ GeoPosition $)$

- GeoPosition: array of latitude and longitude values.

Figure 5.2 - Final setting of our motivational example with cities and crops. The proposed Similarity-aware Division $(\hat{\dot{-}})$ allows us to select cities well suited to produce a given type of crop, taking as input only georeferenced remote sensing images and city bounds.

the discussion in this section, we consider once more our motivational division query about cities and crops. Note that we have been didactically adding complexity to this example in this MSc thesis, from its simplest case that can be solved by the existing operator of division, discussed in the Chapter 1.1 and illustrated in Figures 1.1a, 1.1b and 1.1c (also replicated in Figures 5.1a, 5.1b and 5.1c), up to its final version that we describe as follows and use in the rest of the thesis. From now on, we use an even more realistic setting for this example in which only satellite images (or small tiles extracted from the images, if we receive large images as input) and their corresponding geodetic positions with latitude and longitude values are available to form the dividend relation CityRegions, thus adding complexity to our intermediate setting shown in Figures 5.1d, 5.1e and 5.1f. That is, the final setting of our example does not have 
textual tags to identify the city of each image. Figure 5.2 summarizes this setting. The schema of the dividend CityRegions is given by Sch(CityRegions) = (GeoPosition,Region), in which attribute GeoPosition is an array with the latitude and longitude values that identify the bounds of each image. As an example, for the toy image in Figure 5.3, attribute GeoPosition has the value $([-22.08,47.89],[-21.68,47.89],[-22.08,37.29],[-21.68,37.29])$. The images themselves are in attribute Region. The schema of the divisor Requirements remains unaltered, as in Figure 1.1e, and the quotient relation Cities has obviously an updated schema, i.e., Sch(Cities) $=$ (GeoPosition), with attribute GeoPosition sharing the same domain of its counterpart in CityRegions.

In this setting, the intra-relation comparisons must group the images in CityRegions according to their latitude and longitude coordinates to identify the candidate cities for evaluation and validation of the crop requirements. Note that, besides the images' geodetic positions, the grouping criterion in this example must know the boundaries of each city for which we may have an image, e.g., the boundaries of all Brazilian cities. Also, note that this setting allows a single image to refer to two or more cities, as we illustrate in Figure 5.3. It opens up the following possibilities: (a) the corresponding tuple in CityRegions may be assigned only to the group of city A, since the majority of the image refers to city A; (b) it may be assigned both to the group of city A and to the group of city B, thus allowing the groups to overlap, or; (c) the tuple may be discarded as an outlier that does not help any city to fulfill the crop requirements.

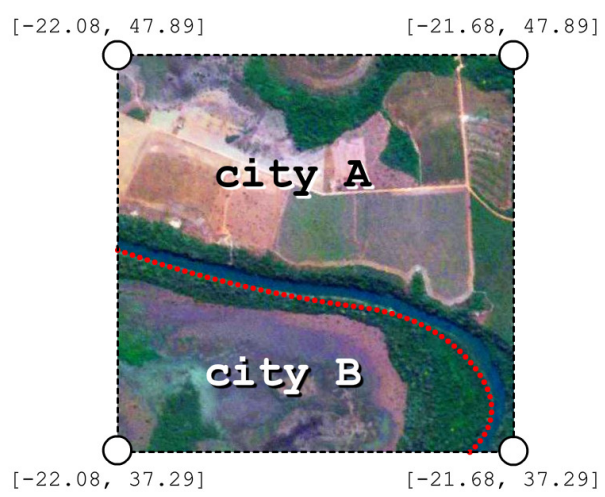

Figure 5.3 - Example image with two cities. Its tuple in the dividend CityRegions may be assigned to the group of city A only, since the majority of the image belongs to city A, or to both the groups of city A and city B. It may also be discarded as an outlier tuple.

Once defined the groups in CityRegions, the inter-relation comparisons must spot the groups that have at least one image similar to each image of the divisor Requirements. As opposed to the intra-relation comparisons, here the images themselves are compared by similarity, regardless of their geodetic positions. For example, let us consider the toy images in Figure 5.4. The city region from Figure 5.4a satisfies the requirement in Figure 5.4b, as they both represent water, so their inter-relation comparison should return TRUE.

The quotient Cities is populated with the groups that satisfy all requirements by 


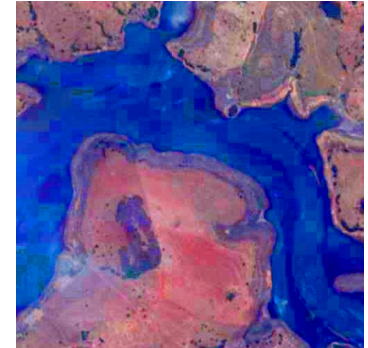

(a) Example city region

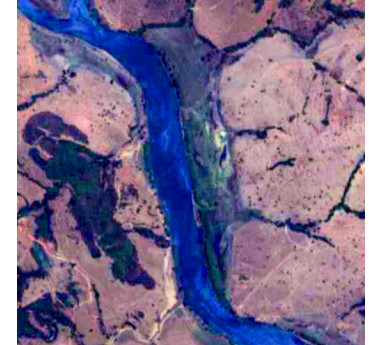

(b) Example crop requirement

Figure 5.4 - Example similar images of water.

similarity, i.e., true inter-relation comparisons with all images in Requirements. We name these groups as valid groups. Note that there exist two possibilities here: (a) to fill Cities with $\pi_{\text {(GeoPosition) }}\left(\mathrm{T}_{G_{k}}\right)$ for each valid group $\mathrm{T}_{G_{k}}$, thus inserting in Cities all distinct values of GeoPosition from these groups, or; (b) to populate Cities with a summarized description of each valid group, like the GeoPosition of the group's most central image or the average latitude and longitude of each corner of the group's images. For brevity and without loss of generality, we considerthe former option in this MSc thesis.

\subsubsection{Formal Definition}

This section formally defines the new Similarity-aware Division $(\hat{\dot{\div}})$ database operator. We first present preliminary definitions. Then, we use them in our main Definition 5.9.

Definition 5.1 - Two relations $\mathrm{T}_{1}$ and $\mathrm{T}_{2}$ are Union compatible if and only if they both have the same number of attributes and each attribute from $\mathrm{T}_{1}$ has the same domain of its counterpart in $\mathrm{T}_{2}$. We consider $A_{i}$ to be the $i^{\text {th }}$ attribute in the schema $S c h(\mathrm{~T})$ of a relation $\mathrm{T}$. The domain of $A_{i}$ is $\operatorname{Dom}\left(A_{i}\right)$. Any two relations $\mathrm{T}_{1}$ and $\mathrm{T}_{2}$ are Union compatible if and only if:

$$
\begin{array}{r}
\left(\left|\operatorname{Sch}\left(\mathrm{T}_{1}\right)\right|=\left|\operatorname{Sch}\left(\mathrm{T}_{2}\right)\right|\right) \wedge \\
\left(\forall A_{i} \in \operatorname{Sch}\left(\mathrm{T}_{1}\right), \forall A_{j} \in \operatorname{Sch}\left(\mathrm{T}_{2}\right), i=j: \operatorname{Dom}\left(A_{i}\right)=\operatorname{Dom}\left(A_{j}\right)\right)
\end{array}
$$

Definition 5.2 - The similarity of attribute values $(\hat{=})$ is represented as $a_{1} \hat{=} a_{2}$, in which $a_{1} \in$ $A_{1}$ and $a_{2} \in A_{2}$. Attributes $A_{1}$ and $A_{2}$ must follow the same metric space $M=\langle\mathbb{S}, d\rangle$ with $\mathbb{S}$ being the data domain and $d: \mathbb{S} \times \mathbb{S} \rightarrow \mathbb{R}^{+}$the distance function, so that $\mathbb{S}=\operatorname{Dom}\left(A_{1}\right)=\operatorname{Dom}\left(A_{2}\right)$ and $a_{1}, a_{2} \in \mathbb{S}$. For a threshold $\xi$, values $a_{1}$ and $a_{2}$ are similar if and only if:

$$
a_{1} \hat{=} a_{2} \Leftrightarrow d\left(a_{1}, a_{2}\right) \leq \xi
$$

Definition 5.3 - The similarity of tuples $(\hat{=})$ is represented as $t_{1} \hat{=} t_{2}$, in which $t_{1}$ and $t_{2}$ are tuples from relations $\mathrm{T}_{1}$ and $\mathrm{T}_{2}$, respectively. One tuple is similar to another if and only if their 
home relations are Union-compatible, and each attribute of the former has a value that is similar to its counterpart in the latter. We consider $t\left[A_{i}\right]$ to be the value of an attribute $A_{i}$ for a tuple $t$. Formally, the similarity of tuples is defined by:

$$
t_{1} \hat{=} t_{2} \Leftrightarrow \forall A_{i} \in S c h\left(\mathrm{~T}_{1}\right), \forall A_{j} \in S c h\left(\mathrm{~T}_{2}\right), i=j: t_{1}\left[A_{i}\right] \hat{=} t_{2}\left[A_{j}\right]
$$

Definition 5.4 - The set membership by similarity $(\hat{\epsilon})$ is represented as $t \hat{\in} \mathrm{T}_{1}$, in which $\mathrm{T}_{1}$ is a relation and $t \in \mathrm{T}$ is a tuple. $\mathrm{T}_{1}$ and $\mathrm{T}$ must be Union-compatible. Tuple $t$ is an element of $\mathrm{T}_{1}$ by similarity if and only if there exists at least one tuple $t_{j} \in \mathrm{T}_{1}$ that is similar to t. Formally, we have:

$$
t \hat{\in} \mathrm{T}_{1} \Leftrightarrow \exists t_{j} \in \mathrm{T}_{1}: t \hat{=} t_{j}
$$

Following the same idea, $t$ is not an element of $\mathrm{T}_{1}$ by similarity if and only if there is no tuple $t_{j} \in \mathrm{T}_{1}$ that is similar to $t$. Formally, it is given by:

$$
t \hat{\notin} \mathrm{T}_{1} \Leftrightarrow \nexists t_{j} \in \mathrm{T}_{1}: t \hat{=} t_{j}
$$

Definition 5.5 - The Subset by similarity $(\hat{\underline{S}})$ is represented as $\mathrm{T}_{1} \hat{\subseteq} \mathrm{T}_{2}$, in which $\mathrm{T}_{1}$ and $\mathrm{T}_{2}$ are Union-compatible relations. Relation $\mathrm{T}_{1}$ is a subset of $\mathrm{T}_{2}$ by similarity if and only if every tuple $t_{i} \in \mathrm{T}_{1}$ is also an element of $\mathrm{T}_{2}$ by similarity. Formally, its is defined by:

$$
\mathrm{T}_{1} \hat{\subseteq} \mathrm{T}_{2} \Leftrightarrow \forall t_{i} \in \mathrm{T}_{1}: t_{i} \hat{\in} \mathrm{T}_{2}
$$

Definition 5.6 - The Difference by similarity $(\hat{-})$ is a binary operation represented as $\mathrm{T}_{1} \hat{-} \mathrm{T}_{2}=$ $\mathrm{T}_{R}$, in which $\mathrm{T}_{1}$ and $\mathrm{T}_{2}$ are Union-compatible relations. The resulting relation $\mathrm{T}_{R}$ has all tuples of $\mathrm{T}_{1}$ that are not members of $\mathrm{T}_{2}$, by similarity. Formally, we have:

$$
\mathrm{T}_{R}=\left\{t_{i}: t_{i} \in \mathrm{T}_{1} \wedge t_{i} \hat{\notin} \mathrm{T}_{2}\right\}
$$

Definition 5.7 - A Group of similars $\mathrm{T}_{G_{k}}$ is a subset of a given relation $\mathrm{T}_{1}$, such that each of its tuples is similar to at least one other tuple in the group, taking into account only a subset of attributes $\mathrm{L} \subseteq S$ ch $\left(\mathrm{T}_{1}\right)$. Relation $\mathrm{T}_{G_{k}}$ is also considered to be a group of similars if $\left|\mathrm{T}_{G_{k}}\right|=1$. Formally, $\mathrm{T}_{G_{k}}$ is a group of similars if and only if:

$$
\begin{array}{r}
\left(\mathrm{T}_{G_{k}} \subseteq \mathrm{T}_{1}\right) \wedge \\
\left(\left(\forall t_{i} \in \mathrm{T}_{G_{k}}:\left(\exists t_{j} \in \mathrm{T}_{G_{k}}, i \neq j: t_{i}[\mathrm{~L}] \hat{=} t_{j}[\mathrm{~L}]\right)\right) \vee\left(\left|\mathrm{T}_{G_{k}}\right|=1\right)\right)
\end{array}
$$


Definition 5.8 - One Similarity grouping $\mathrm{T}_{G}$ is the set of all groups of similars extracted from a relation $T_{1}$, taking into account a subset of attributes $L \subseteq S c h\left(T_{1}\right)$. The number of groups is $\mathrm{\kappa}=\left|\mathrm{T}_{G}\right|$. Formally, we have:

$$
\mathrm{T}_{G}=\left\{\mathrm{T}_{G_{i}}: \mathrm{T}_{G_{i}} \text { is a group of similars from } \mathrm{T}_{1} \text { regarding } \mathrm{L}\right\}
$$

The restriction as follows applies:

$$
\mathrm{T}_{1}=\bigcup_{k=1}^{\kappa} \mathrm{T}_{G_{k}}
$$

Definition 5.9 - The Similarity-aware division $(\hat{\dot{-}})$ is a binary operation represented as:

$\mathrm{T}_{1}\left[\mathrm{~L}_{1} \hat{\dot{\div}} \mathrm{L}_{2}\right] \mathrm{T}_{2}=\mathrm{T}_{R}$, in which $\mathrm{T}_{1}, \mathrm{~T}_{2}$ and $\mathrm{T}_{R}$ are relations that respectively correspond to the dividend, the divisor and the quotient. $\mathrm{L}_{1} \subseteq \operatorname{Sch}\left(\mathrm{T}_{1}\right)$ and $\mathrm{L}_{2} \subseteq \operatorname{Sch}\left(\mathrm{T}_{2}\right)$ are lists of attributes, so that relations $\pi_{\left(\mathrm{L}_{1}\right)} \mathrm{T}_{1}$ and $\pi_{\left(\mathrm{L}_{2}\right)} \mathrm{T}_{2}$ are Union-compatible. The schema of $\mathrm{T}_{R}$ is defined as $\operatorname{Sch}\left(\mathrm{T}_{R}\right)=\overline{\mathrm{L}_{1}}=\operatorname{Sch}\left(\mathrm{T}_{1}\right)-\mathrm{L}_{1}$. The instance of $\mathrm{T}_{R}$ is the union of $\pi_{\left(\overline{\mathrm{L}_{1}}\right)} \mathrm{T}_{G_{k}}$ for the largest possible number of groups of similars $\mathrm{T}_{G_{k}} \in \mathrm{T}_{G}$, such that $\mathrm{T}_{R} \times \mathrm{T}_{2} \subseteq \mathrm{T}_{1}$. Formally, the quotient $\mathrm{T}_{R}$ is defined as:

$$
\mathrm{T}_{R}=\bigcup_{k=1}^{\kappa} \begin{cases}\pi_{\left(\overline{\mathrm{L}_{1}}\right)} \mathrm{T}_{G_{k}}, & \text { if } \forall t_{j} \in \pi_{\left(\mathrm{L}_{2}\right)}\left(\mathrm{T}_{2}\right):\left(\exists t_{i} \in \pi_{\left(\mathrm{L}_{1}\right)}\left(\mathrm{T}_{G_{k}}\right): t_{i} \hat{=} t_{j}\right) \\ \varnothing, & \text { otherwise. }\end{cases}
$$

The remainder of the similarity-aware division is given by:

$$
\mathrm{T}_{1} \hat{-} \mathrm{T}_{R} \times \mathrm{T}_{2}
$$

\subsection{Proposed Algorithms}

This section presents two novel algorithms that we carefully developed for the similarityaware division. They are detailed in Algorithms 2 and 3. We focused on distinct possible configuration settings: (a) Algorithm 2 suits for the case when the dividend $T_{1}$ has index structures, i.e., Metric Access Methods - MAM, ready to be used for the attributes in list $L_{1},-$ it can be seen as the similarity-aware version of our Algorithm 1 from Chapter 4; (b) Algorithm 3 is our proposal for the cases when these indexes do not exist. Both Algorithms 2 and 3 are fast and scalable; their time complexities are $O\left(\left|\mathrm{~T}_{2}\right| \log \left|\mathrm{T}_{1}\right|\right)$ and $O\left(\left|\mathrm{~T}_{1}\right| \log \left|\mathrm{T}_{2}\right|\right)$ respectively. They work very efficiently with large dividend and quotient relations that do not fit in main memory; note that the cardinality of the divisor $T_{2}$ is tiny in nearly all of the practical uses for the division, since division queries with too many requirements are very likely to be useless, by returning empty results. 
One crucial observation must be made here. We decided to separate the task of grouping/clustering the tuples of the dividend from the rest of the division, and developed algorithms to perform the latter part only. We made this decision since grouping/clustering refers to a broad, well-stablished research area with well-known algorithms that significantly vary depending on the data domain, the field of application, the amount of data, and many other environmental-based variations that affect the result of the division. For example, let us consider again our running example with cities and crops illustrated in Figure 5.2. As we discussed in Section 5.1.2, a single image in this setting may refer to two or more cities, like in our illustration in Figure 5.3, so the corresponding tuple from relation CityRegions may be assigned to the group of city A only, since most of the image belongs to city A, it may alternativelly be assigned to both the group of city A and to the group of city B, or it may even be discarded as an outlier tuple that does not help any city to fulfill the crop requirements. Decisions of this nature depend exclusively on the application, and not on the division itself; nevertheless, they obviously affect the query results. For the sake of generality, the algorithms developed in this MSc work are independent of the strategy adopted for grouping/clustering; they receive the results of this task as an input parameter.

Our algorithms have five input parameters: $\mathrm{T}_{1}, \mathrm{~L}_{1}, \mathrm{~T}_{2}, \mathrm{~L}_{2}$ and $\mathrm{T}_{G}$. Parameters $\mathrm{T}_{1}$ and $\mathrm{T}_{2}$ are the dividend and the divisor, in that order. $L_{1}$ and $L_{2}$ respectively identify the attributes of relations $T_{1}$ and $T_{2}$ to be compared with each other, thus defining how to validate the candidate groups with regard to the requirements. For each pair of attributes for comparison, it must be informed the metric to be used to measure similarity and the similarity threshold $\xi$. In our current implementation, this information is provided together with the attributes in parameter $L_{1}$. Finally, parameter $\mathrm{T}_{G}$ identifies the group $\mathrm{T}_{G_{k}}$ (or the groups) that each tuple of relation $\mathrm{T}_{1}$ belongs to.

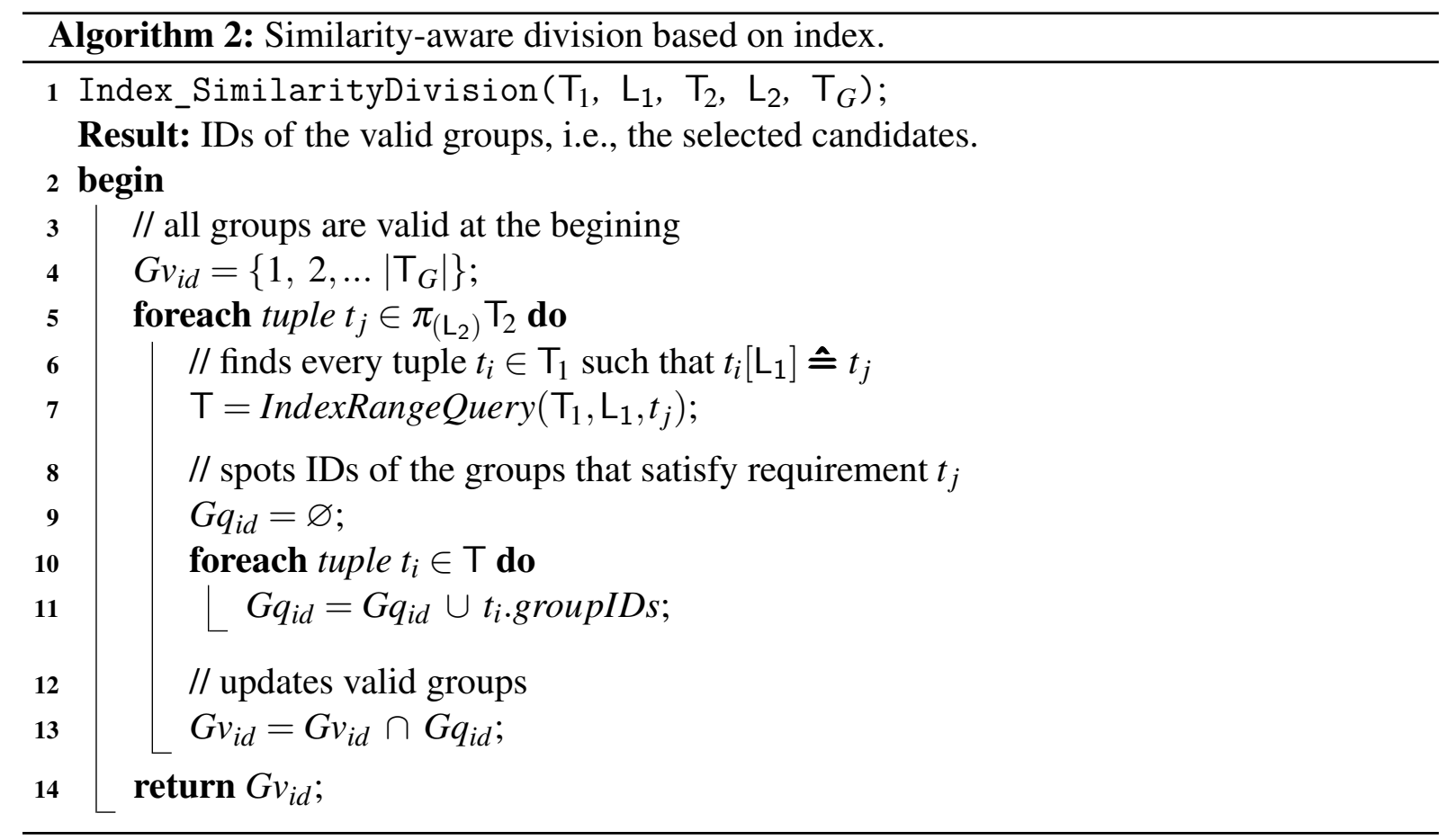


Algorithm 2 is the pseudocode of the first solution that we propose. Here, we assume that relation $T_{1}$ has MAM indexes ready to be used for the attributes in $L_{1}$. The algorithm works by iteratively updating a set $G v_{i d}$ with valid groups' identifiers. All candidate groups are considered to be valid at the beginning, so the set starts with $G v_{i d}=\left\{1,2, \ldots\left|\mathrm{T}_{G}\right|\right\}$. Then, for each requirement $t_{j}$ from $\mathrm{T}_{2}$, we perform a range query in $\mathrm{T}_{1}$ using the requirement itself as the query center and taking advantage of the existing indexes to speed-up the execution. The query finds every tuple $t_{i} \in \mathrm{T}_{1}$ such that $t_{i}\left[\mathrm{~L}_{1}\right] \stackrel{\wedge}{=} t_{j}$. The next step is to build a set $G q_{i d}$ with all identifiers of the groups that satisfy requirement $t_{j}$, that is, the groups of the tuples returned by the query. Then, set $G v_{i d}$ is updated with the intersection of the valid groups from the previous iteration and the groups that meet the current requirement. At the end of the execution, only identifiers of candidate groups that meet all the requirements remain in $G v_{i d}$.

Time complexity: Algorithm 2 performs $\left|T_{2}\right|$ range queries in relation $T_{1}$. State-of-theart Metric Access Methods allow us to perform each range query in $O\left(\log \left|\mathrm{T}_{1}\right|\right)$ time. Therefore, the total runtime of Algorithm 2 is $O\left(\left|\mathrm{~T}_{2}\right| \log \left|\mathrm{T}_{1}\right|\right)$.

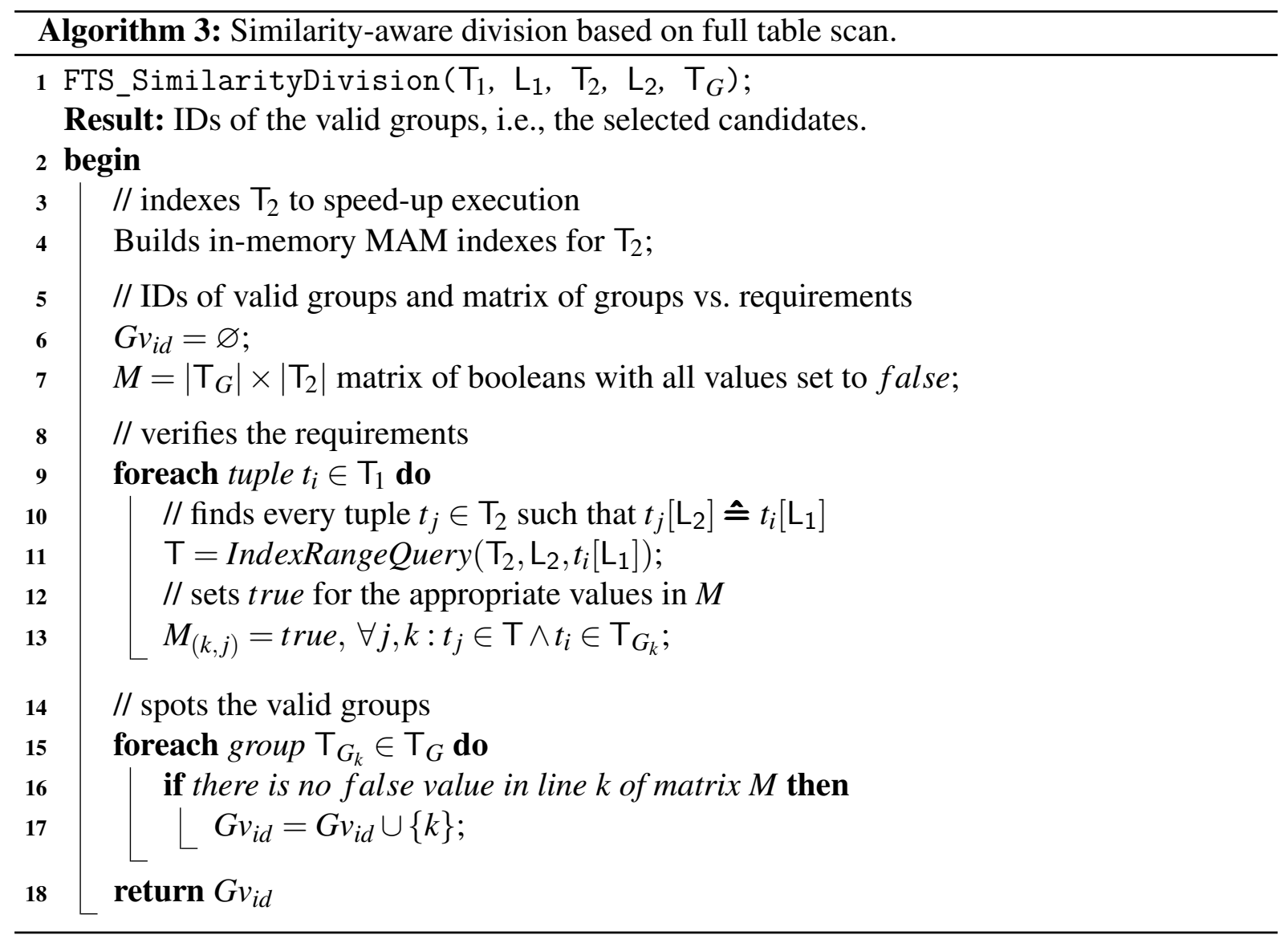

Algorithm 3 is our proposed solution for the cases in which the dividend $T_{1}$ does not have MAM indexes ready to be used for the attributes in $L_{1}$. As we discussed in the first paragraph of this section, we must be able to deal with large relations $T_{1}$ that do not fit in main memory. On the other hand, the cardinality of the divisor $T_{2}$ is tiny in nearly all of the practical uses for the division, so it is safe to assume that main memory is enough to store indexes for $T_{2}$. With that 
in mind, our proposal in Algorithm 3 is to perform only sequential disk accesses by building in-memory MAM indexes for $T_{2}$, and running a full table scan on $T_{1}$ to query the requirements fulfilled by each of its tuples. Here, we use a matrix $M$ of $\left|T_{G}\right| \times\left|T_{2}\right|$ boolean values to indicate which requirements are fulfilled by each group of $T_{1}$. Initially, no group satisfies any requirement, that is, all values in $M$ are false. For each tuple of $T_{1}$, we then perform a range query on $T_{2}$ using the tuple itself as the query center and taking advantage of the indexes to speed-up the queries. Note that only the indexes in main memory are used here; we do not reload pages of $T_{2}$ from disk to perform the queries. The results obtained represent requirements fulfilled, so they are used to update matrix $M$. Once $\mathrm{T}_{1}$ is read, the algorithm builds a set $G v_{i d}$ with identifiers of the valid groups; a group $\mathrm{T}_{G_{k}} \in \mathrm{T}_{G}$ is valid if and only if there is no false value in line $k$ of matrix $M$. Set $G v_{i d}$ is then returned to the user.

Time complexity: Algorithm 3 indexes the divisor $\mathrm{T}_{2}$ in $O\left(\left|\mathrm{~T}_{2}\right|\right)$ time. Then it runs $\left|\mathrm{T}_{1}\right|$ range queries in relation $T_{2}$. State-of-the-art Metric Access Methods allow us to perform each range query in $O\left(\log \left|\mathrm{T}_{2}\right|\right)$ time. Therefore, Algorithm 3 takes $\left|\mathrm{T}_{2}\right|$ plus $\left|\mathrm{T}_{1}\right| \log \left|\mathrm{T}_{2}\right|$ units of time to run. Since the former is much smaller than the latter, the overall cost is essentially $O\left(\left|\mathrm{~T}_{1}\right| \log \left|\mathrm{T}_{2}\right|\right)$.

\section{Conclusion}

In this chapter, we presented the main contribution of this MSc work: the new similarityaware division database operator, including its design, formal definition, and two novel algorithms well suited to execute the operator in a fast and scalable manner. The next chapter, presents the experiments that we executed to verify the scalability and the efficiency of our algorithms, and it also reports results of two case studies performed to validate the usability of the new operator in real world applications. 

This chapter describes a comprehensive set of experiments performed to evaluate our similarity-aware division. We validated the semantics and the usability of our proposals by studying two distinct applications of high-impact: (a) agricultural support through the analysis of remote sensing images, following our motivational example with cities and crops, and; (b) selective animal breeding for better milk production supported by the analysis of genetic data. Our techniques were also tested with synthetic data. Specifically, we aimed at answering two main questions:

1. How accurate is the similarity-aware division in the sense of returning what the users expect to receive?

2. How effective and scalable are the algorithms that we propose?

The experiments were performed in a machine with an Intel ${ }^{\circledR}$ Core ${ }^{\mathrm{TM}}$ i7 processor of $2.67 \mathrm{GHz}$ and $8 G B$ of RAM. Algorithms 2 and 3 were implemented in $\mathrm{C}++$ with page buffer management. Library Arboretum ${ }^{1}$ was used for the indexed range queries.

\subsubsection{Agricultural Support}

We validated the semantics of the new operator in a case study with geopositioned remote sensing images to semi-automatically identify cities well-suited to produce particular types of crops. The following Brazilian cities were studied: Sapezal-MT, Sorriso-MT, AnhangueraGO, Catolândia-BA and Volta Redonda-RJ. The images come from satellite RapidEye ${ }^{2}$ with size $5 k \times 5 k$ pixels. Each pixel represents a region of 5 squared meters with values for the spectral bands Red, Green, Blue, Red Edge and Near Infrared. This particular dataset was kindly provided to us by researchers from the Centre of Meteorological and Climate Research Applied 


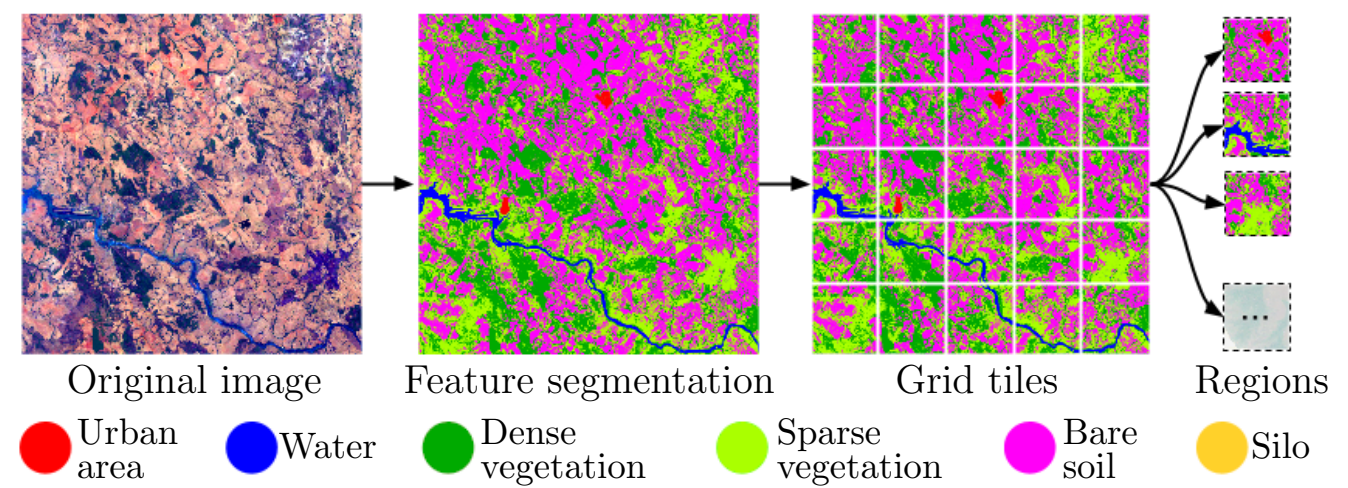

Figure 6.1 - Pipeline to preprocess remote sensing images for our case study in agriculture. Best viewed in color.

to Agriculture - CEPAGRI at the University of Campinas - UNICAMP, Brazil, and the Brazilian Agricultural Research Corporation - EMBRAPA.

(a) Silo

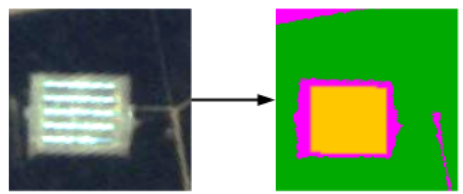

(b) Bare soil

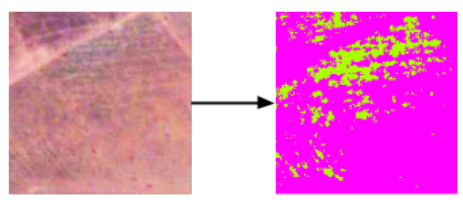

(c) Water

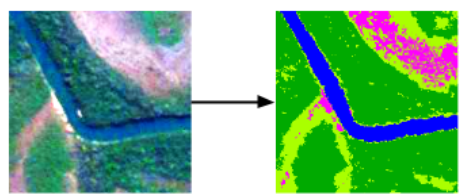

(d) Urban area

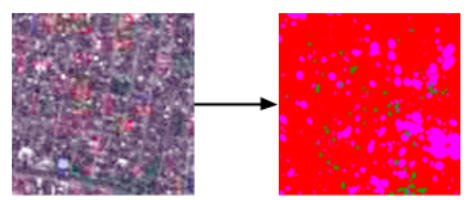

Urban
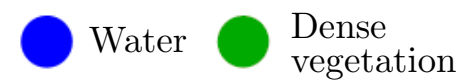

Sparse
vegetation

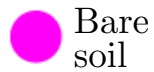

Silo

Figure 6.2 - The images used in the divisor for the case study in agriculture. They represent the requirements of the crop production. Best viewed in color.

We adopted the schema of our motivational example shown in Figure 5.2. Figure 6.1 illustrates how we preprocessed the data to populate relations CityRegions and Requirements. At first, each original image was segmented in software QGIS $^{3}$ based on a semi-supervised classification performed by algorithm Spectral Angle Mapping (SOHN; REBELLO, 2002). Six classes were considered: urban area, water, dense vegetation, sparse vegetation, bare soil and silo. The images were then partitioned into equal-sized tiles of $100 \times 100$ pixes each. The resulting image tiles compose relation CityRegions. Relation Requirements was built to represent four 
basic needs of a crop production: (a) silo infrastructure for storage; (b) bare soil for the plantation; (c) water for irrigation, and; (d) urban areas to support the production. Figure 6.2 shows the exact tiles used to represent each of these requirements. The Earth Mover's distance (RUBNER; TOMASI; GUIBAS, 2000) was employed to compare images of crop requirements with tiles in CityRegions. The similarity threshold was set to 0.05 .

We ran the similarity-aware division on these data, and found out that city Sorriso-MT is the only one that successfully satisfies all four requirements. The other cities were able to satisfy some of the requirements, but not all of them. Figure 6.3 provides a visual description for part of this result. As we can see, city Sorriso-MT has indeed all four requirements. On the other hand, city Anhanguera-MT fails to provide silo infrastructure. Similar results were obtained for the other cities that we studied; they are omitted for brevity.

Thanks to the proposed similarity-aware division, we were able to semi-automatically analyze the cities' infrastructure required to produce a crop, taking as input geopositioned remote sensing images and city boundaries. These results indicate that our new operator may help (i) to estimate crop productivity; (ii) to support financial and/or governmental institutions to decide whether or not to loan money to producers; (iii) to support governmental strategies to preserve the environment, still maximizing the productivity, and the like. Note that very little human intervention was necessary, i.e., one single image tile per requirement was enough to evaluate the candidate cities for the crop production, and, as our algorithms are fast and scalable, the new similarity-aware division is potentially useful in coutrywide analyses, and even in broader analyses.

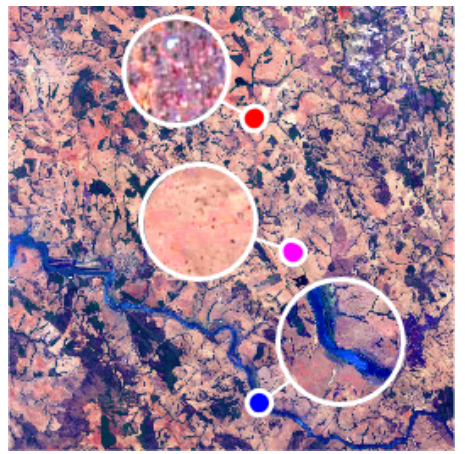

(a) City Anhanguera-MT: the Silo requirement is missing.

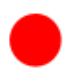
Urban area

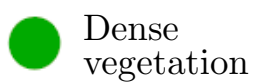

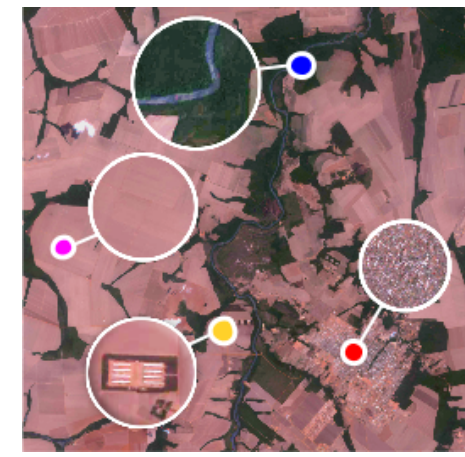

(b) City Sorriso-MT: it has all four requirements.

$$
\begin{aligned}
& \text { Sparse } \\
& \text { vegetation } \\
& \text { soil }
\end{aligned}
$$

Silo

Figure 6.3 - Example result for two cities. Best viewed in color.

\subsubsection{Selective Animal Breeding}

This section presents the second case study that we performed to validate our proposals, now focused on selective animal breeding for better milk production. It uses as a basis the background concepts on genetic data that were previously presented in Section 2.4 from Chapter 
2. We first discuss how to use the original operator of division to query these data. Then, we show how our similarity-aware division improves the usability of the query, according to the needs of geneticists, and report experimental results.

\section{Relational Division on Genetic Data}

The original operator of division can analyze genetic data to select individuals with specific characteristics. Let us use the query in Table 6.1 as an example. The query refers to a problem of "candidate elements and requirements" aimed at selecting animals well-evaluated for breeding, e.g., according to their ability to produce milk. The dividend relation contains SNPs representing variations in the DNA sequences of candidate animals. In the example, the animals are identified by attribute ID. Each animal has several tuples storing its SNPs - attributes Allele 1 and Allele 2 together with the corresponding positions along the chromosomes. Table 6.1a illustrates three candidates for selective breeding. The genetic conditions desired for each candidate are in Table $6.1 \mathrm{~b}$. The resulting relation in Table $6.1 \mathrm{c}$ has the animals that attend all requirements, in this case only the animal with $I D=1$ is selected.

Table 6.1 - Example of the division used to select animals that satisfy desired genetic conditions, e.g., exceptional milk producers. The dataset represents SNP alleles and their positions along the chromosomes of candidate animals. As opposed to our similarity-aware division, the original division operator fails to identify animal 3, despite the fact that it has SNPs similar to all requirements.

\begin{tabular}{|c|c|c|c|c|}
\hline \multicolumn{2}{|r|}{$\overline{\mathrm{L}_{1}}$} & \multicolumn{3}{|c|}{$\mathrm{L}_{1}$} \\
\hline \multirow{4}{*}{$\mathrm{T}_{G_{1}}$} & ID & Pos & Allele 1 & Allele 2 \\
\hline & 1 & 0.10 & 1 & 2 \\
\hline & 1 & 44.5 & 2 & 2 \\
\hline & 1 & 84.0 & 2 & 1 \\
\hline \multirow{3}{*}{$\mathrm{T}_{G_{2}}$} & 2 & 0.10 & 2 & 2 \\
\hline & 2 & 44.0 & 2 & 1 \\
\hline & 2 & 83.0 & 1 & 2 \\
\hline \multirow{3}{*}{$\mathrm{T}_{G_{3}}$} & 3 & 0.10 & 1 & 2 \\
\hline & 3 & 44.5 & 1 & 1 \\
\hline & 3 & 84.5 & 2 & 1 \\
\hline
\end{tabular}

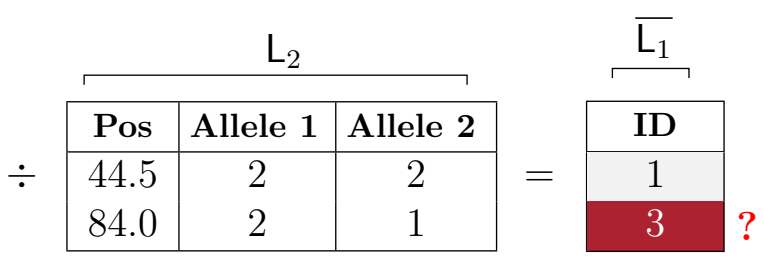
(b) $\mathrm{T}_{2}-$ Desired genetic conditions.
(c) $T_{R}-$
Selected animals.

(a) $T_{1}$ - Genetic data of candidate animals.

\section{Similarity-aware Division on Genetic Data}

As we can see in the previous example, the original operation of division is very useful to express complex queries for genetic data with a single operator. However, the same division operation cannot properly answer the query if we need to compare the data following a criterion of similarity, instead of identity. A much more realistic use for this dataset is to compare the position of the alleles along the chromosome by similarity, using a distance function. Thus, we could set a threshold of distance to consider if a SNP is similar to another without being exactly the same. Actually, this approach is commonly mandatory in genetic analyses (BALDING, 2006), because 
several polymorphisms with the same behavior are likely to appear near along the chromosome. Also, geneticists commonly need to analyze only the zygosity of the SNPs, that is, if both alleles in a SNP have the same value, then they are homozygous, otherwise they are classified as heterozygous. Thanks to our similarity-aware division, one can define functions to measure these kinds of similarity and use it to validate the desired genetic conditions without modifying the dataset. For example, let us consider as similar homozygous SNPs positioned within a radius 1 of distance; and use the same idea to classify heterozygous SNPs. In this case, the division query in Table 6.1 would also return the animal with $I D=3$, since its SNPs $\langle 44.5,1,1\rangle$ and $\langle 84.5,2,1\rangle$ are similar to the requirements $\langle 44.5,2,2\rangle$ and $\langle 84.0,2,1\rangle$, respectively.

We performed a case study using the similarity-aware division to select animals that have predefined genetic conditions, represented by SNPs. The dataset used is the QTL-MAS Workshop ${ }^{4}$ public data, in which a cattle population has been simulated. It is a well-known dataset commonly used in the community of genetic analysis that contains 10,000 SNP genotypes for 4,100 animals, leading to a dividend relation with more than $\mathbf{4 0}$ million tuples. The dataset also provides the True Breeding Value for each animal, which we used as the ground truth to evaluate the results of our queries. The True Breeding Value of an animal quantifies its ability to develop desired production traits due to its genetic conditions, in this case, referring to milk production. This ability depends on SNPs that are part of the genome of each animal. Only very few SNPs are directly related to the development of some valuable trait, and, due to the linkage disequilibrium (MOORE; ASSELBERGS; WILLIAMS, 2010), these influences are not restricted to exactly the same SNP in each animal, but also to certain neighboring regions, thus forcing geneticists to deal with similarity comparisons.

We performed several queries using the proposed similarity-aware division to select animals that fulfill by similarity a set of requirements for top-quality milk production. The results showed that it is possible to identify animals very well-evaluated for breeding that are not identical in all genetic characteristics, nevertheless, they share similar regions of SNPs responsible for the development of the desired trait. For that, we selected the animal with the best True Breeding Value - let us name it as the ideal animal - and used its SPNs as the desired genetic conditions in the divisor. We used a deviation of 0.1 mega base pairs $(\mathrm{Mb})$ in the threshold of distance, which is equivalent to a radius of two SNPs, as well as the zygosity of the alleles as the criterion of similarity. Our similarity-aware division was able to select animals that do not have parent-child relationship to the ideal animal in any degree, and yet they are almost as good as it is. In comparison, the traditional division did not retrieve any animal for the same queries, without the similarity criteria.

Thanks to the proposed similarity-aware division, we were able to semi-automatically analyze the animals' abilities to become top-quality milk producers, taking only SNPs as input. These results indicate that our new operator may help (i) producers to perform selective breeding;

4 http://qtl-mas-2012.kassiopeagroup.com/ 
(ii) geneticists to better understand the consequences of DNA polymorphisms; (iii) governmental institutions to teach producers how to increase their productivity and/or to decide whether or not to loan money to them, besides a variety of other potential applications. Note that very little human intervention was necessary, as a single outstanding animal was used to evaluate

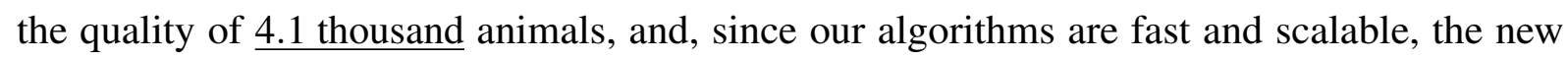
similarity-aware division is potentially useful even in broader analyses.

\subsubsection{Synthetic Data}

We created synthetic datasets to represent possible input relations for the similarityaware division. They were used to evaluate the performance and the scalability of our proposed Algorithms 2 Index_SimilarityDivision and 3 FTS_SimilarityDivision, from Chapter 5. The dividend relation has two attributes in all datasets; each attribute belongs to one of the lists $L_{1}$ and $\overline{L_{1}}$ from Definition 5.9. The attribute in $\overline{L_{1}}$ has a traditional data type, i.e., short text. List $L_{1}$ has a complex attribute; each of its values is in fact a feature vector of 5 numbers. The divisor has always an attribute with the same characteristics of the dividend's complex attribute. It belongs to list $L_{2}$. We used the Euclidian distance (WILSON; MARTINEZ, 1997) to perform similarity comparisons between feature vectors from the dividend and those in the divisor.

In our datasets, the dividend size varies from thousands to millions of tuples. The size of the divisor goes from 5 up to one hundred tuples. Note that we did not create larger divisor relations since division queries with too many requirements are very likely to be useless, by returning empty results. Unless otherwise specified, each dataset has 10 candidate groups with similar cardinalities in the dividend, and the data was configured to have $10 \%$ of the groups satisfying all of the divisor's requirements.

Figure 6.4a reports the algorithms' runtime with increasing dividend cardinalities in a $\log -\log$ plot. The size of the divisor was fixed in five tuples for this experiment. Note that the index-based algorithm is tens of times faster than the one that uses a full table scan, but both of our algorithms scaled linearly in runtime regarding the dividend cardinality.

Figure $6.4 \mathrm{~b}$ reports in a log-log plot the results obtained with increasing sizes for the divisor. The dividend size was fixed in one million tuples for this experiment. Again, the indexbased approach was considerably faster than running a full table scan. Nevertheless, note that the latter algorithm scaled sub-linearly in this experiment, while the former one presented a linear trend.

Figure $6.4 \mathrm{c}$ reports in a log-log plot the performance of our algorithms when the number of groups increases. No significant variation in runtime was noticed for the algorithms, but the index-based approach was slightly more sensitive to this parameter when the number of groups got close to 10 thousands, compared with the other approach. The quotient relation has $10 \%$ of the groups in all datasets used. 
Figure $6.4 \mathrm{~d}$ reports the performance of our algorithms when we increase the percentage of groups that satisfy all requirements, i.e., the valid groups, with regard to the total number of groups in the dividend. The total number of groups was fixed to one thousand for this experiment. The algorithms were tested with as little as $1 \%$ of groups adequate to the requirements up to $50 \%$ of valid groups. No significant variation in runtime was noticed.

Figure 6.4e reports the performance of our algorithms in datasets with unbalanced group sizes. For this experiment we created three datasets with equal-sized dividend relations storing 500 thousand tuples each, but used distinct strategies to assign the tuples to groups in each dataset, so that the group sizes follow a normal, uniform or exponential distribution. As we can see, unbalanced group sizes do not lead to relevant variations in runtime.

Figure $6.4 \mathrm{f}$ reports the performance of our algorithms with varying similarity threshold configurations. For clarity, we plot threshold values as percentages of the dataset's diameter. That is, the value 0 leads to the identity comparison while 100 specifies that all pairs of points in the dataset are similar to each other. As expected, the runtime of our algorithms grows as we soften the similarity criterion, but this increase is considerably small up to a very reasonable threshold of $25 \%$ of the dataset's diameter.

\subsection{Conclusion}

This chapter presented two case studies performed to validate our proposals in supporting real applications of high-impact: crop production and animal selective breeding. We also described experiments performed to evaluate the efficiency of our algorithms using synthetic data with input and output relations containing up to millions of tuples. Our algorithms presented either linear or sub-linear scalability tendencies for all datasets studied, thus enabling their usage with very large amounts of data, and also corroborating the corresponding theoretical time complexity analyses presented in Chapter 5. In the next chapter, we discuss the generality of the similarity-aware division bys describing how it can be helpful for three additional applications. 
- Index_SimilarityDivision _-FTS_SimilarityDivision _ Linear scalability

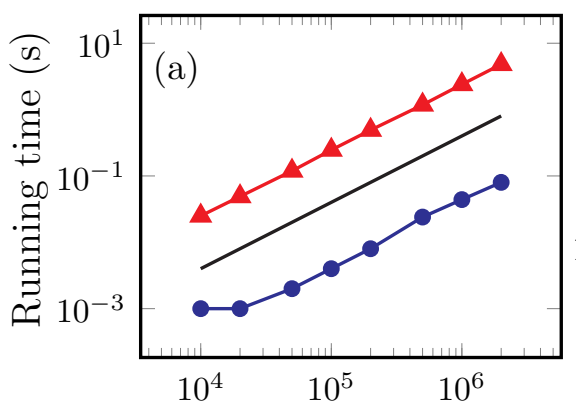

Dividend Size
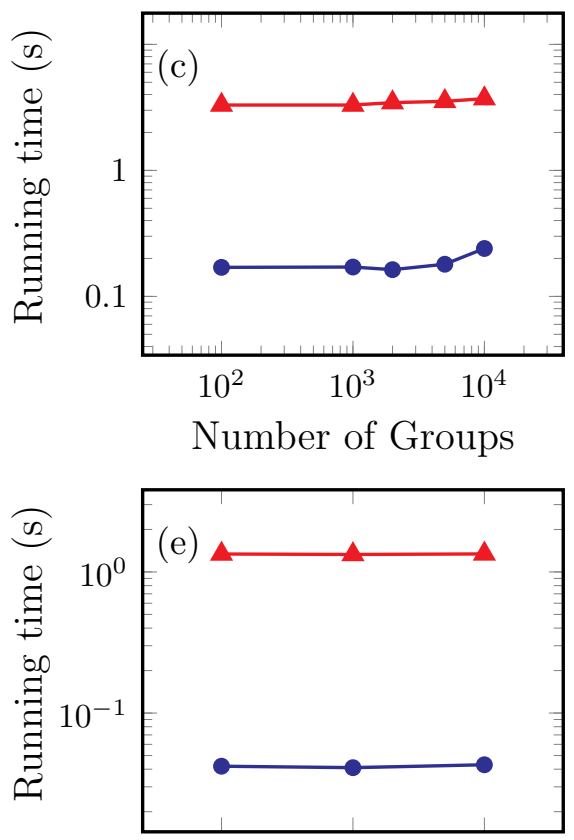

Normal Uniform Exp.

Group Size Distribution

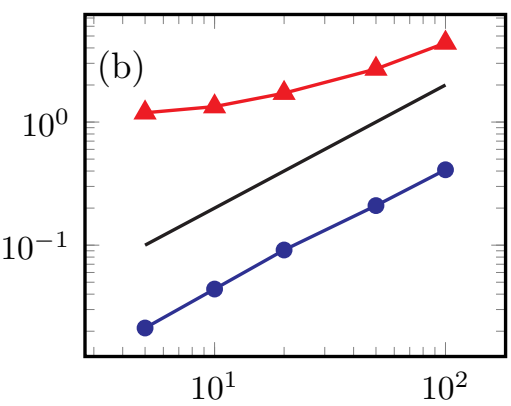

Divisor Size
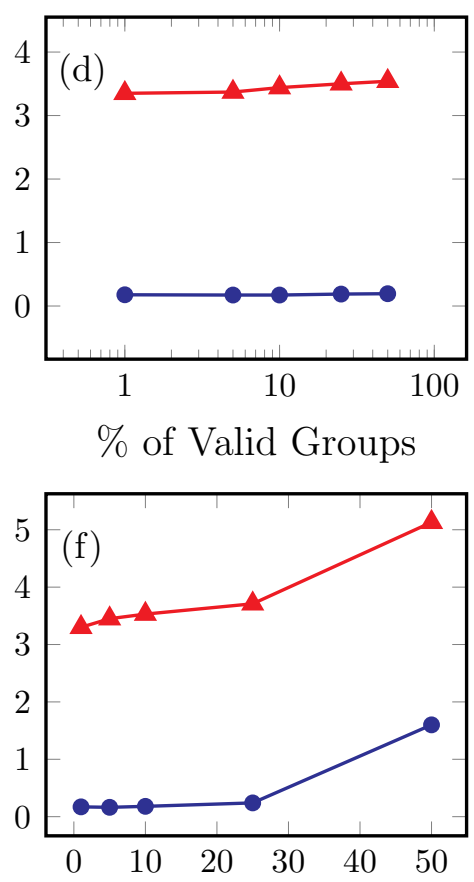

Similarity Threshold (\%)

Figure 6.4 - Runtime measurements for our algorithms in synthetic data. 


\section{DISCUSSION}

The previous chapter described how we used the similarity-aware division in two real applications of high-impact: agriculture and genetics. Here, we provide evidences in support of the new operator's generality by describing how it can be helpful in three additional applications.

\subsubsection{Automatic Quality Control in Industry}

Let us consider any industrial assembly line for which the manager needs to deploy an automatic quality control system to work in real-time. To make it possible, he/she could select one "ideal unity" of the type of product produced in the line, collect images of this unity from several angles and zoom levels, and store these images in a relation IdealUnity with schema $S c h$ (IdealUnity) = (Image). For each new unity produced, images in the same angles and zoom levels could be collected, and stored in a relation NewUnits with schema Sch(NewUnits) = (UnitId, Image). In this setting, the similarity-aware division, represented by NewUnits $[($ Image $) \hat{\div}$ (Image) $]$ IdealUnit = ApprovedUnits, could verify the quality of the newly produced units automatically. The output relation ApprovedUnits would have identifiers of the units approved in the test, i.e., units with images similar to all the corresponding images from the "ideal unit";

\subsubsection{Digital Library Search}

Let us consider any digital library with hundreds of thousands of books, magazines, papers, among others, and the need to search out documents according to their content. In such libraries, a frequent type of search takes as input a set of terms defined by the user, i.e., words or expressions, and outputs documents that include all of these terms. For example, a query for documents related to commercial aviation in the USA may have the terms "airplane", "enterprise" and "USA". Note that an ideal result for this query should probably contain not only the documents with these exact three terms, but also documents with terms simi- 
lar to the user-defined ones. For example, a document with terms "Boeing 787", "United Airlines" and "Chicago" should be retrieved. Comparing terms by similarity would also prevent issues related to typing errors that may occur in the input data and/or in the documents, like when the term "ariplane" is mistakenly used instead of "airplane". It is interesting to note that the similarity-aware division is a seamless way to implement this query in RDBMS. To make it possible, one could use a relation Documents to store the most relevant terms of each document and represent the query terms in a relation Input. The schemas would be $S c h($ Documents $)=($ DocumentId, Term $)$ and $S c h($ Input $)=($ Term $)$. In this setting, the division Documents $[($ Term $) \hat{\div}($ Term $)]$ Input $=$ Output would answer the query by selecting the documents with terms similar to all terms provided by the user.

\subsubsection{Prospective Client Identification in Enterprises}

Many public institutions around the world are required by law to buy products and services following a very strict protocol: they must make public calls for bids with textual descriptions of each item to be bought. An auction-like procedure is commonly followed in such a way that any enterprise can make a bid, as long as it is able to provide all items of the call. For example, several of such calls start everyday in Brazil, many of them referring to hundreds of items each. The manual identification of calls for which a given enterprise can make a bid is therefore costly and time consuming. Nevertheless, it is interesting to note that the similarity-aware division may help to automatize this process. Let us assume the existence of a crawler application that analyzes governmental websites, constantly extracting item descriptions from active calls for bids. Once it finds a new call, the corresponding descriptions form a relation $\mathrm{Call}$ with schema $\mathrm{Sch}(\mathrm{Call})=$ (Item). In this setting, any group of enterprises could take advantage of the similarity-aware division to identify prospective clients, i.e., public institutions that may buy their products and services. Assuming that a relation GroupItems stores textual descriptions for the products and services provided by the group, with schema $S c h$ (GroupItems) = (EnterpriseId, Item), the division operation GroupItems $[($ Item $) \hat{\div}($ Item $)] \mathrm{Call}=$ ApprovedEnterprises retrieves the enterprises of the group that are able to make bids. An enterprise is returned if and only if it has item descriptions similar to all descriptions in the call. Note that similarity comparisons are essential in this application, since public calls must be free of bias; the item descriptions in these calls cannot name brands, registered trademarks, and the like, so it is virtually impossible for them to be identical to the descriptions of real products.

\subsection{Conclusion}

In this chapter, we provided evidences to support the generality of the new similarityaware division operator by describing how it can be helpful for three additional applications. The next chapter concludes this MSc thesis with a brief description of the work performed, a 
discussion on its main contributions and a list of publications generated. 

In this MSc work we identified severe limitations on the usability of the Relational Division to process complex data, and tackled the problem by extending it into the new Similarityaware Division $(\hat{\div})$ database operator. As opposed to the existing division, our new operator is naturally well suited to answer queries with an idea of "candidate elements and exigencies" to be performed on complex data from real applications of high-impact, such as the numerous modern applications that process images, genetic data, audio, long texts, fingerprints, and several other "non-traditional" data types that must be compared by similarity. For example, we demonstrated in case studies that the similarity-aware division can support genetics and agriculture, and we also discussed how it may be helpful in digital library search, industrial quality control and even to identify prospective clients for enterprises.

We formally defined the similarity-aware division and carefully designed two fast and scalable algorithms for it. The first approach takes advantage of MAM index structures to speedup the queries; the second one uses a full table scan for the cases when appropriate indexes are not available. To evaluate the efficiency of our algorithms, we performed experiments in synthetic data with input and output relations containing up to millions of tuples. In these experiments our algorithms always presented either linear or sub-linear scalability tendencies, thus enabling their usage with very large amounts of data. Theoretical time complexity analyses were also provided for both algorithms.

To validate our proposals, we performed case studies on the support of agriculture and genetics through semi-automatic complex data analysis. First, the similarity-aware division allowed us to identify cities well-suited to produce a particular type of crop, based on the analysis of geopositioned remote sensing images. We studied satellite imagery from Brazilian cities, considering each city as a candidate to produce the crop. Once defined the crop production needs, our new operator answered the query accordingly, retrieving the only city that satisfies all requirements. Visual inspection corroborates the accuracy of this result. In the second case study, we selected animals with predefined genetic conditions represented by Single Nucleotide 
Polymorphisms - SNPs. We were particularly interested in supporting selective animal breeding for better milk production. The requirements were defined as the SNPs of an ideal animal, that is, the overall best milk producer. Thanks to our similarity-aware division, we were able to select other animals that produce almost as much milk as the ideal animal and do not have parent-child relationships with it in any degree from a dataset with 4.1 thousand animals described by more than 40 million SNPs. Ground truth data available for this dataset corroborates the accuracy of our results. Note that very little human intervention was necessary in both case studies that we performed: (a) one image tile per requirement was enough to evaluate the candidate cities for the crop production, and; (b) a single outstanding animal allowed us to suggest other animals for selective breeding. Provided that our algorithms are fast and scalable, we argue that the new similarity-aware division is potentially useful to analyze very large amounts of complex data, even in real-time.

To support the aforementioned work, we also studied well-known strategies used to implement the traditional division in RDBMS, and proposed a new algorithm that is potentially faster than these approaches. A novel data generator for the division was also developed.

The work performed during this MSc program generated four publications:

1. André S. Gonzaga, Robson L. F. Cordeiro: A New Division Operator to Handle Complex Objects in Very Large Relational Datasets. EDBT 2017: 474-477 (International Conference Qualis A1);

2. André S. Gonzaga, Robson L. F. Cordeiro: The Similarity-aware Relational Division Database Operator. ACM SAC, 2017: 913-914 (International Conference - Qualis A1);

3. André S. Gonzaga, Robson L. F. Cordeiro: Fast and Scalable Relational Division on Database Systems. SBBD 2016: 169-174;

4. Paulo H. Oliveira, Antonio C. Fraideinberze, Natan A. Laverde, Hugo Gualdron, André S. Gonzaga, Lucas D. Ferreira, Willian D. Oliveira, José F. Rodrigues Jr., Robson L. F. Cordeiro, Caetano Traina Jr., Agma J. M. Traina, Elaine P. M. de Sousa: On the Support of a Similarity-enabled Relational Database Management System in Civilian Crisis Situations. ICEIS 2016: 119-126 (International Conference - Qualis B2).

An improved and expanded version of the article published at EDBT 2017 is currently being evaluated for publication at the Elsevier Information Systems Journal (Qualis A2). 
BALDING, D. J. A tutorial on statistical methods for population association studies. Nat. Rev. Genet., v. 7, n. 10, p. 781-791, 2006. Cited on page 56.

BARIONI, M. C. N.; RAZENTE, H. L.; TRAINA, A. J. M.; Traina Jr., C. Seamlessly integrating similarity queries in SQL. Softw., Pract. Exper., v. 39, n. 4, p. 355-384, 2009. Cited 2 times on pages 20 and 33.

BELOHLAVEK, R.; VYCHODIL, V. Query systems in similarity-based databases: Logical foundations, expressive power, and completeness. In: Proc. of the 2010 ACM Symposium on Applied Computing. New York, NY, USA: ACM, 2010. (SAC '10), p. 1648-1655. ISBN 978-1-60558-639-7. Disponível em: <http://doi.acm.org/10.1145/1774088.1774444>. Cited 2 times on pages 20 and 33 .

BOSC, P.; PIVERT, O. A new semantics for the division of fuzzy relations in relational databases. In: Proceedings of the EUSFLAT-ESTYLF Joint Conference, Palma de Mallorca, Spain, September 22-25, 1999. [S.l.: s.n.], 1999. p. 243-246. Cited on page 32.

BUDÍKOVÁ, P.; BATKO, M.; ZEZULA, P. Query language for complex similarity queries. In: ADBIS. [S.1.: s.n.], 2012. p. 85-98. Cited 2 times on pages 20 and 33.

CAMPS, D. High performance relational division in sql server. Simple-Talk, Red Gate, 2014. [Online; acessed April 26,2016]. Disponível em: <https://www.simple-talk.com/sql/ t-sql-programming/divided-we-stand-the-sql-of-relational-division>. Cited 2 times on pages 31 and 32.

CELKO, J. Divided we stand: The sql of relational division. Simple-Talk, Red Gate, 2009. [Online; acessed April 26,2016]. Disponível em: <https://www.simple-talk.com/sql/ t-sql-programming/divided-we-stand-the-sql-of-relational-division $>$. Cited 2 times on pages 31 and 32.

CODD, E. F. Relational completeness of data base sublanguages. In: R. Rustin (ed.): Database Systems: 65-98, Prentice Hall and IBM Research Report RJ 987, San Jose, California, 1972. Cited 4 times on pages 19, 25, 26, and 31 .

The Relational Model for Database Management: Version 2. Boston, MA, USA: Addison-Wesley Longman Publishing Co., Inc., 1990. ISBN 0-201-14192-2. Cited 3 times on pages 19,25 , and 27.

CORDEIRO, R. L. F.; FALOUTSOS, C.; Traina Jr., C. Data Mining in Large Sets of Complex Data. [S.1.]: Springer, 2013. I-XI, 1-116 p. (Springer Briefs in Computer Science). ISBN 978-14471-4889-0. Cited 2 times on pages 20 and 33.

CORDEIRO, R. L. F.; TRAINA, A. J. M.; FALOUTSOS, C.; Traina Jr., C. Halite: Fast and scalable multiresolution local-correlation clustering. IEEE Trans. Knowl. Data Eng., v. 25, n. 2, p. 387-401, 2013. Disponível em: <http://dx.doi.org/10.1109/TKDE.2011.176>. Cited on page 33 . 
FITZ, P. R. Geoprocessamento sem complicação. São Paulo, SP, Brazil: Oficina de Textos, 2008. Cited on page 27.

GALINDO, J.; MEDINA, J. M.; GARRIDO, M. C. Fuzzy division in fuzzy relational databases: an approach. Fuzzy Sets and Systems, v. 121, n. 3, p. 471-490, 2001. Disponível em: <https: //doi.org/10.1016/S0165-0114(99)00156-6>. Cited on page 32.

GALINDO, J.; URRUTIA, A.; PIATTINI, M. Fuzzy Databases: Modeling, Design and Implementation: Modeling, Design and Implementation. [S.1.]: Idea Group, 2005. 1-320 p. ISBN 1-59140-324-3. Cited on page 32.

GARCIA-MOLINA, H.; ULLMAN, J. D.; WIDOM, J. Database systems - The Complete Book. Upper Saddle River, NJ, USA: Pearson Education, 2002. 189-237 p. ISBN 978-0-13098043-4. Cited on page 25.

GONZAGA, A.; CORDEIRO, R. Fast and scalable relational division on database systems. In: Proc. of the 31th Brazilian Symposium on Databases. [S.1.]: Brazilian Computer Society, 2016. p. 169-173. ISBN 978-85-7669-342-0. Cited on page 31.

JACOX, E. H.; SAMET, H. Metric space similarity joins. ACM Trans. Database Syst., ACM, New York, NY, USA, v. 33, n. 2, p. 7:1-7:38, jun. 2008. ISSN 0362-5915. Disponível em: $<$ http://doi.acm.org/10.1145/1366102.1366104>. Cited 2 times on pages 20 and 33.

KALASHNIKOV, D. V. Super-ego: Fast multi-dimensional similarity join. The VLDB Journal, Springer-Verlag New York, Inc., Secaucus, NJ, USA, v. 22, n. 4, p. 561-585, ago. 2013. ISSN 1066-8888. Disponível em: <http://dx.doi.org/10.1007/s00778-012-0305-7>. Cited 3 times on pages 20, 22, and 33 .

LEINDERS, D.; BUSSCHE, J. Van den. On the complexity of division and set joins in the relational algebra. In: ACM. Proceedings of the twenty-fourth ACM SIGMOD-SIGACTSIGART symposium on Principles of database systems. [S.1.], 2005. p. 76-83. Cited on page 31 .

LI, W.; GODZIK, A. Cd-hit: a fast program for clustering and comparing large sets of protein or nucleotide sequences. Bioinformatics, Oxford Univ Press, v. 22, n. 13, p. 1658-1659, 2006. Cited on page 33.

MARRI, W. J. A.; MALLUHI, Q.; OUZZANI, M.; TANG, M.; AREF, W. G. The similarityaware relational intersect database operator. In: Similarity Search and Applications. [S.1.]: Springer, 2014. p. 164-175. Cited 3 times on pages 20, 22, and 33.

The similarity-aware relational database set operators. Inf. Syst., Elsevier Science Ltd., Oxford, UK, UK, v. 59, n. C, p. 79-93, jul. 2016. ISSN 0306-4379. Disponível em: <http: //dx.doi.org/10.1016/j.is.2015.10.008>. Cited 3 times on pages 20, 22, and 33 .

MATOS, V. M.; GRASSER, R. Assessing performance of the relational division operator. Data Base Management, CRC Press, v. 22-20-30, p. 1-11, 2001. Cited on page 31.

MAULIK, U.; BANDYOPADHYAY, S.; MUKHOPADHYAY, A. Multiobjective Genetic Algorithms for Clustering: Applications in Data Mining and Bioinformatics. [S.1.]: Springer Science \& Business Media, 2011. Cited on page 33. 
MEDINA, J. M.; PONS, O.; MIRANDA, M. A. V. GEFRED: A generalized model of fuzzy relational databases. Inf. Sci., v. 76, n. 1-2, p. 87-109, 1994. Disponível em: <https://doi.org/10. 1016/0020-0255(94)90069-8>. Cited on page 32.

MOORE, J. H.; ASSELBERGS, F. W.; WILLIAMS, S. M. Bioinformatics challenges for genome-wide association studies. Bioinformatics, v. 26, n. 4, p. 445-455, Feb 2010. Cited on page 57.

POLA, I. R.; CORDEIRO, R. L.; JR, C. T.; TRAINA, A. J. A new concept of sets to handle similarity in databases: The simsets. In: Similarity Search and Applications. [S.1.]: Springer, 2013. p. 30-42. Cited 2 times on pages 20 and 33.

POLA, I. R. V.; CORDEIRO, R. L. F.; JR., C. T.; TRAINA, A. J. M. Similarity sets: A new concept of sets to seamlessly handle similarity in database management systems. Inf. Syst., v. 52, p. 130-148, 2015. Disponível em: <http://dx.doi.org/10.1016/j.is.2015.01.011>. Cited 3 times on pages 20,22 , and 33 .

RUBNER, Y.; TOMASI, C.; GUIBAS, L. J. The earth mover's distance as a metric for image retrieval. International Journal of Computer Vision, v. 40, n. 2, p. 99-121, 2000. ISSN 15731405. Disponível em: <http://dx.doi.org/10.1023/A:1026543900054>. Cited on page 55.

SANTOS, L. F. D.; OLIVEIRA, W. D.; FERREIRA, M. R. P.; TRAINA, A. J. M.; TRAINA JR., C. Parameter-free and domain-independent similarity search with diversity. In: Proceedings of the 25th International Conference on Scientific and Statistical Database Management. New York, NY, USA: ACM, 2013. (SSDBM), p. 5:1-5:12. ISBN 978-1-4503-1921-8. Disponível em: <http://doi.acm.org/10.1145/2484838.2484854>. Cited 2 times on pages 22 and 33.

SILVA, Y.; AREF, W.; LARSON, P.-A.; PEARSON, S.; ALI, M. Similarity queries: their conceptual evaluation, transformations, and processing. The VLDB Journal, Springer-Verlag, v. 22, n. 3, p. 395-420, 2013. ISSN 1066-8888. Disponível em: <http://dx.doi.org/10.1007/ s00778-012-0296-4>. Cited 3 times on pages 20, 22, and 33.

SILVA, Y. N.; ALY, A. M.; AREF, W. G.; LARSON, P.-A. Simdb: a similarity-aware database system. In: SIGMOD Conference. [S.1.: s.n.], 2010. p. 1243-1246. Cited 2 times on pages 20 and 33.

SILVA, Y. N.; AREF, W. G.; ALI, M. H. Similarity group-by. In: IOANNIDIS, Y. E.; LEE, D. L.; NG, R. T. (Ed.). Proceedings of the 25th International Conference on Data Engineering, ICDE 2009, March 292009 - April 2 2009, Shanghai, China. IEEE Computer Society, 2009. p. 904-915. ISBN 978-0-7695-3545-6. Disponível em: <http://dx.doi.org/10.1109/ICDE.2009. $113>$. Cited 2 times on pages 22 and 33.

The similarity join database operator. In: IEEE. Data Engineering (ICDE), 2010 IEEE 26th International Conference on. [S.1.], 2010. p. 892-903. Cited 3 times on pages 20, 22, and 33 .

SILVA, Y. N.; PEARSON, S. Exploiting database similarity joins for metric spaces. Proc. VLDB Endow., VLDB Endowment, v. 5, n. 12, p. 1922-1925, ago. 2012. ISSN 2150-8097. Disponível em: <http://dx.doi.org/10.14778/2367502.2367538>. Cited 3 times on pages 20, 22, and 33.

SILVA, Y. N.; PEARSON, S. S.; CHON, J.; ROBERTS, R. Similarity joins: Their implementation and interactions with other database operators. Inf. Syst., v. 52, p. 149-162, 2015. Disponível em: <http://dx.doi.org/10.1016/j.is.2015.01.008>. Cited 3 times on pages 20, 22, and 33. 
SOHN, Y.; REBELLO, N. Supervised and unsupervised spectral angle classifiers. Photogrammetric Engineering and Remote Sensing, v. 68, n. 12, p. 1271-1280, 2002. Cited on page 54.

TAMANI, N.; LIETARD, L.; ROCACHER, D. A relational division based on a fuzzy bipolar r-implication operator. In: FUZZ-IEEE 2013, IEEE International Conference on Fuzzy Systems, Hyderabad, India, 7-10 July, 2013, Proceedings. [s.n.], 2013. p. 1-7. Disponível em: $<$ https://doi.org/10.1109/FUZZ-IEEE.2013.6622331>. Cited on page 32.

TANG, M.; TAHBOUB, R. Y.; AREF, W. G.; ATALLAH, M. J.; MALLUHI, Q. M.; OUZZANI, M.; SILVA, Y. N. Similarity group-by operators for multi-dimensional relational data. IEEE Trans. Knowl. Data Eng., v. 28, n. 2, p. 510-523, 2016. Disponível em: <http://dx.doi.org/10. 1109/TKDE.2015.2480400>. Cited 3 times on pages 20, 22, and 33.

. In: 32nd IEEE International Conference on Data Engineering, ICDE 2016, Helsinki, Finland, May 16-20, 2016. IEEE Computer Society, 2016. p. 1448-1449. ISBN 978-1-5090-2020-1. Disponível em: <http://dx.doi.org/10.1109/ICDE.2016.7498368>. Cited 2 times on pages 22 and 33.

WILSON, D. R.; MARTINEZ, T. R. Improved heterogeneous distance functions. Journal of Artificial Intelligence Research, AI Access Foundation, USA, v. 6, n. 1, p. 1-34, 1997. ISSN 1076-9757. Disponível em: <http://dl.acm.org/citation.cfm?id=1622767.1622768>. Cited on page 58.

ZEZULA, P.; AMATO, G.; DOHNAL, V.; BATKO, M. Similarity Search - The Metric Space Approach. [S.1.]: Kluwer, 2006. v. 32. 1-191 p. (Advances in Database Systems, v. 32). ISBN 978-0-387-29146-8. Cited on page 20.

ZHANG, K.; QIN, Z. S.; LIU, J. S.; CHEN, T.; WATERMAN, M. S.; SUN, F. Haplotype block partitioning and tag snp selection using genotype data and their applications to association studies. Genome Research, Cold Spring Harbor Lab, v. 14, n. 5, p. 908-916, 2004. Cited on page 28 . 